ESAIM: M2AN 46 (2012) 559-593

DOI: $10.1051 / \mathrm{m} 2 \mathrm{an} / 2011060$
ESAIM: Mathematical Modelling and Numerical Analysis

www.esaim-m2an.org

\title{
INITIAL-BOUNDARY VALUE PROBLEMS FOR SECOND ORDER SYSTEMS OF PARTIAL DIFFERENTIAL EQUATIONS*
}

\author{
Heinz-Otto Kreiss ${ }^{1}$, Omar E. Ortiz ${ }^{2}$ and N. Anders Petersson ${ }^{3}$
}

\begin{abstract}
We develop a well-posedness theory for second order systems in bounded domains where boundary phenomena like glancing and surface waves play an important role. Attempts have previously been made to write a second order system consisting of $n$ equations as a larger first order system. Unfortunately, the resulting first order system consists, in general, of more than $2 n$ equations which leads to many complications, such as side conditions which must be satisfied by the solution of the larger first order system. Here we will use the theory of pseudo-differential operators combined with mode analysis. There are many desirable properties of this approach: (1) the reduction to first order systems of pseudo-differential equations poses no difficulty and always gives a system of $2 n$ equations. (2) We can localize the problem, i.e., it is only necessary to study the Cauchy problem and halfplane problems with constant coefficients. (3) The class of problems we can treat is much larger than previous approaches based on "integration by parts". (4) The relation between boundary conditions and boundary phenomena becomes transparent.
\end{abstract}

Mathematics Subject Classification. 35L20, 65M30.

Received August 31, 2009.

Published online January 11, 2012.

\section{INTRODUCTION}

The theory for first order hyperbolic systems, which was developed with fluid problems in mind, is by now rather well understood. It turned out that energy estimates via 'integration by parts' and characteristics are the most important ingrediencies in the theory.

Second order hyperbolic systems often describe problems where wave propagation is dominant. In bounded domains this leads to a large number of boundary phenomena like glancing waves and surface waves. Attempts have previously been made to write a second order system consisting of $n$ equations as a larger first order system.

\footnotetext{
Keywords and phrases. Well-posed 2nd-order hyperbolic equations, surface waves, glancing waves, elastic wave equation, Maxwell equations.

* This work performed under the auspices of the U.S. Department of Energy by Lawrence Livermore National Laboratory under Contract DE-AC52-07NA27344. O.E.O. acknowledges support by grants 05/B415 and 214/10 from SeCyT-Universidad Nacional de Córdoba, 11220080100754 from CONICET, PICT17-25971 from ANPCYT, and the Partner Group grant of the Max Planck Institute for Gravitational Physics, Albert-Einstein-Institute (Germany).

1 Träsko-Storö Institute of Mathematics, NADA, KTH, 10044 Stockholm, Sweden. hokreiss@nada.kth.se

2 Facultad de Matemáticas, Astronomía y Física and IFEG, Universidad Nacional de Córdoba, Ciudad Universitaria, CP:X5000HUA, Córdoba, Argentina.

3 Center for Applied Scientific Computing, Lawrence Livermore National Laboratory, Livermore, California, USA.
} 
However, boundary phenomena such as glancing and surface waves correspond to generalized eigenvalues which are not handled by the theory for first order systems. Furthermore, the resulting first order system often consists of more than $2 n$ equations, which leads to many complications. In particular, the first order system must in general be augmented by side conditions to guarentee that solutions of the first order system satisfy the original second order system.

In this paper we describe a theory for second order hyperbolic systems based on Laplace and Fourier transform, with particular emphasis on boundary processes corresponding to generalized eigenvalues. Our theory uses pseudo-differential operators combined with mode analysis, and builds upon the theory for first order systems developed in $[3,4]$. This approach has many desirable properties: (1) once a second order system has been Laplace and Fourier transformed it can always be written as a system of $2 n$ first order pseudo-differential equations. Therefore, the theory of $[3,4]$ also applies here. (2) We can localize the problem, i.e., it is only necessary to study the Cauchy problem and halfplane problems with constant coefficients. (3) The class of problems we can treat is much larger than previous approaches based on "integration by parts". (4) The relation between boundary conditions and boundary phenomena becomes transparent.

The remainder of the paper is organized as follows. In Section 2 we state the general problem and provide some basic definitions. In Section 3 we treat in detail the fundamental problem of a single wave equation in a half-plane subject to different types of boundary conditions. In Section 4 we first study two wave equations coupled through the boundary conditions and then outline a theory for the general case of $n$ second order wave equations. This theory proves that all essential difficulties already occur for scalar wave equations coupled through the boundary conditions. Numerical experiments are presented in Section 5, where we study the different classes of boundary phenomena for two wave equations coupled through the boundary conditions.

\section{INITIAL-BOUNDARY VALUE PROBLEMS FOR SECOND ORDER HYPERBOLIC SYSTEMS}

\subsection{Well posed problems}

In this paper we want to consider second order systems which are of the form

$$
u_{t t}=P_{0}(D) u+F(x, t), \quad t \geq 0, \quad x \in \Omega, \quad F \in \mathrm{C}_{0}^{\infty}(\Omega),
$$

in the halfspace $\Omega=\left\{x_{1} \geq 0,-\infty<x_{j}<\infty, j=2, \ldots, r\right\}$. Here

$$
P_{0}(D)=A_{1} D_{1}^{2}+\sum_{j=2}^{r} B_{j} D_{j}^{2},
$$

where

$$
A_{1}=A_{1}^{*}>0, B_{j}=B_{j}^{*}>0,
$$

are $n \times n$ constant matrices, $u$ is a vector valued function with $n$ components and we are using the notation

$$
\begin{aligned}
x & =\left(x_{1}, \ldots, x_{r}\right), \quad D=\left(D_{1}, \ldots, D_{r}\right), D_{j}=\partial / \partial x_{j}, \\
u_{t} & =\partial u / \partial t=D_{t} u, \quad u_{x_{j}}=D_{j} u .
\end{aligned}
$$

At $t=0$ we give initial conditions by

$$
u(x, 0)=f_{1}(x), \quad u_{t}(x, 0)=f_{2}(x) .
$$

We are interested in smooth solutions which belong to $\mathrm{L}^{2}(\Omega)$ and satisfy, at the boundary $\Gamma=\left\{x_{1}=0,-\infty<\right.$ $\left.x_{j}<\infty, j=2, \ldots, r\right\} n$ linearly independent boundary conditions.

$$
L u=: C_{0} u_{t}+\sum_{j=1}^{r} C_{j} u_{x_{j}}=g, \quad g \in \mathrm{C}_{0}^{\infty}(\Gamma) .
$$


Here $C_{0}, C_{j}$ are constant $n \times n$ matrices, $C_{1}$ is non-singular and, without loss of generality, we assume it to be normalised

Assumption 2.1. $C_{1}=I$.

To facilitate the use of Laplace transformation in time, we frequently assume that the initial data are homogeneous, i.e., $f_{1}=f_{2} \equiv 0$. This is however no restriction, since it is always possible to change variables in a problem with general initial data such that the initial data becomes homogeneous in the new variable. Since the Cauchy problem is well posed (see Sect. 4.2) we can extend the definition of the forcing and the initial data smoothly to the whole of $\mathbb{R}^{r}(x)$ and determine its solution. Then we subtract this solution from the halfplane problem and obtain a new halfplane problem where only the boundary data do not vanish. This is a very natural procedure because all the difficulties and many physical phenomena arise at the boundary.

We now introduce some key definitions that classify the problems according to estimates one can achieve.

Definition 2.2. Consider (2.1)-(2.3) for $F=0, f_{1}=f_{2}=0$. The problem is called strongly boundary stable if there are constants $\eta_{0} \geq 0$, and $K>0$ which are independent of $g$, such that for all $\eta>\eta_{0} \geq 0, T \geq 0$

$$
\int_{0}^{T} \mathrm{e}^{-2 \eta t}\left(\|u(\cdot, t)\|_{\mathrm{H}^{1}(\Gamma)}^{2}+\left\|u_{t}(\cdot, t)\right\|_{\mathrm{H}^{0}(\Gamma)}^{2}\right) \mathrm{d} t \leq K \int_{0}^{T} \mathrm{e}^{-2 \eta t}\|g(\cdot, t)\|_{\mathrm{H}^{0}(\Gamma)}^{2} \mathrm{~d} t .
$$

Definition 2.3. The problem (2.1)-(2.3) is called boundary stable if there are constants $\eta_{0} \geq 0, K>0$ and $\alpha>0$, which are independent of $g$, such that for all $\eta>\eta_{0}, T \geq 0$,

$$
\int_{0}^{T} \mathrm{e}^{-2 \eta t}\|u(\cdot, t)\|_{\mathrm{H}^{0}(\Gamma)}^{2} \mathrm{~d} t \leq \frac{K}{\eta^{\alpha}} \int_{0}^{T} \mathrm{e}^{-2 \eta t}\|g(\cdot, t)\|_{\mathrm{H}^{0}(\Gamma)}^{2} \mathrm{~d} t .
$$

Here $\|u\|_{\mathrm{H}^{p}}^{2}$ denotes the norm composed of the $\mathrm{L}^{2}$-norm of $u$ and all its derivatives up to order $p$. Thus (2.4) tells us that we "gain" one derivative while (2.5) says that $u$ is as smooth as the data. The constants $\alpha, \eta_{0}$ are very important. If $\eta_{0}=0$, then we can choose $\eta=\frac{1}{T}$ for every fixed $T>0$. This shows that the solution grows at most like $T^{\alpha}$ with time. If $\eta_{0}>0$, then there is bounded exponential growth. This can happen when lower order terms are present.

The boundary estimates allow us also to obtain interior estimates. In Section 3.2 we will prove

Theorem 2.4. Consider (2.1)-(2.3) with $F=0$. If the problem is boundary stable, then we obtain interior estimates of the form $(2.4),(2.5)$ where $\|u(\cdot, t)\|_{\mathrm{H}^{0}(\Gamma)}^{2}$ is replaced by $\|u(\cdot, t)\|_{\mathrm{H}^{0}(\Omega)}^{2}$ and $\alpha$ by $\tilde{\alpha} \geq \alpha+1$, respectively. If the problem is not boundary stable, then it is illposed.

Since we can always reduce the data such that only $g \neq 0$, we could restrict ourselves to this case. However, we are interested in differential equations with variable coefficients in general domains. Thus we have also to discuss the case that $F \neq 0$. In particular, we have to show that the problem is stable against perturbations by lower order (first order) terms of the differential equations.

Definition 2.5. The problem (2.1)-(2.3) with $f_{1}=f_{2}=0$ is called strongly stable if there exists $\eta_{0} \geq 0, T \geq 0$ and $K>0$, which are independent of $g$ and $F$ such that, for all $\eta>\eta_{0}$,

$$
\begin{gathered}
\eta \int_{0}^{T} \mathrm{e}^{-2 \eta t}\left(\|u(\cdot, t)\|_{\mathrm{H}^{1}(\Gamma)}^{2}+\left\|u_{t}(\cdot, t)\right\|_{\mathrm{H}^{0}(\Gamma)}^{2}\right) \mathrm{d} t+\eta^{2} \int_{0}^{T} \mathrm{e}^{-2 \eta t}\left(\|u(\cdot, t)\|_{\mathrm{H}^{1}(\Omega)}^{2}+\left\|u_{t}(\cdot, t)\right\|_{\mathrm{H}^{0}(\Omega)}^{2}\right) \mathrm{d} t \\
\leq K\left[\eta \int_{0}^{T} \mathrm{e}^{-2 \eta t}\|g(\cdot, t)\|_{\mathrm{H}^{0}(\Gamma)}^{2} \mathrm{~d} t+\int_{0}^{T} \mathrm{e}^{-2 \eta t}\|F(\cdot, t)\|_{\mathrm{H}^{0}(\Omega)}^{2} \mathrm{~d} t\right] .
\end{gathered}
$$


Clearly, if (2.6) holds, then the problem is strongly boundary stable. For first order systems the classical theory (see $[3,4]$ ) tells us that also the converse is true: if the problem is strongly boundary stable, then it is strongly stable. As we will see, after Laplace and Fourier transformation we can write our problem again as a first order system, which satisfies all the conditions of the classical theory, and therefore the results of that theory are also valid for second order systems. In particular, the problem is stable against lower order perturbations both for the differential equations and the boundary conditions. (See appendix of [5]).

Due to physical phenomena like glancing and surface waves, the problems for second order systems are often only boundary stable. This leads to

Definition 2.6. We call the problem (2.1)-(2.3) stable if it is boundary stable and if, for $g=0$, there exists $\eta_{0} \geq 0, K>0$ and $\alpha>0$ which are independent of $F$ such that, for all $\eta>\eta_{0}$,

$$
\int_{0}^{T} \mathrm{e}^{-2 \eta t}\left(\|u(\cdot, t)\|_{\mathrm{H}^{1}(\Omega)}^{2}+\left\|u_{t}(\cdot, t)\right\|_{\mathrm{H}^{0}(\Omega)}^{2}+\|u(\cdot, t)\|_{\mathrm{H}^{0}(\Gamma)}^{2}\right) \mathrm{d} t \leq \frac{K}{\eta^{\alpha}} \int_{0}^{T} \mathrm{e}^{-2 \eta t}\|F(\cdot, t)\|_{\mathrm{H}^{0}(\Omega)}^{2} \mathrm{~d} t .
$$

If $(2.7)$ holds, then we can obtain an estimate even when $g \neq 0$. We split the problem into two; one with $g=0$ and $F \neq 0$ and the other with $g \neq 0$ and $F=0$. For the first problem we obtain (2.7) and for the other we use Theorem 2.4.

In applications there is often a standard energy estimate, which can be obtained by integration by parts provided that $g=0$. This estimate can be written as

$$
\|u(\cdot, t)\|_{\mathrm{H}^{1}(\Omega)}^{2}+\left\|u_{t}(\cdot, t)\right\|_{\mathrm{H}^{0}(\Omega)}^{2} \leq K\left[\|u(\cdot, 0)\|_{\mathrm{H}^{1}(\Omega)}^{2}+\left\|u_{t}(\cdot, 0)\right\|_{\mathrm{H}^{0}(\Omega)}^{2}+\int_{0}^{t}\|F(\cdot, \tau)\|_{\mathrm{H}^{0}(\Omega)}^{2} d \tau\right],
$$

where the constant $K$ is independent of $F$. In this case we need only to show that the problem is boundary stable.

Theorem 2.7. The problem is stable if it is boundary stable and, for $g=0$, the energy estimate (2.8) holds.

One might be tempted to replace the requirement (2.7) by the weaker estimate

$$
\int_{0}^{T} \mathrm{e}^{-2 \eta t}\left(\|u(\cdot, t)\|_{\mathrm{H}^{0}(\Omega)}^{2}+\|u(\cdot, t)\|_{\mathrm{H}^{0}(\Gamma)}^{2}\right) \mathrm{d} t \leq \frac{K}{\eta^{\alpha}} \int_{0}^{T} \mathrm{e}^{-2 \eta t}\|F(\cdot, t)\|_{\mathrm{H}^{0}(\Omega)}^{2} \mathrm{~d} t .
$$

However, the definition is not stable against lower order perturbations. In Section 3.3 we will give an example which is algebraically unstable, i.e., with time the solution loses more and more derivatives.

Definition 2.8. We call the problem (2.1)-(2.3) unstable if the estimate (2.7) does not hold.

For first order systems the generalization to variable coefficients (and then to quasilinear equations) uses the theory of pseudo-differential operators and requires the construction of a symmetrizer, as described in [3], which is smooth in all variables. If the problem is strongly boundary stable, then, as we have mentioned above, the same construction can be used for second order systems. If the problem is only boundary stable, then we have to modify the construction. This can be done but is technically somewhat complicated and the details are beyond the scope of this paper. However, we will make the result plausible.

Since the stability against lower order perturbations is crucial for the generalization of our results to systems with variable coefficients in general domains, we shall give a proof in Section 2.2.

It is also well known that stability against lower order perturbations allows us to use "localization" to decompose an initial boundary value problem on a general compact domain into a finite number of problems that are either initial values problems in the whole space, or initial boundary value problems in the half space. We illustrate the technique with a simple example in one dimension.

Consider the initial boundary value problem for the wave equation on the strip

$$
u_{t t}=u_{x x}, \quad x \in[0,1], \quad t \in[0, \infty)
$$


with initial and boundary conditions

$$
u(x, 0)=f_{1}(x), \quad u_{t}(x, 0)=f_{2}(x), \quad B_{0} u(0, t)=g_{0}(t), \quad B_{1} u(1, t)=g_{1}(t),
$$

where $B_{0}$ and $B_{1}$ are linear first order differential operators.

A partition of unity of $[0,1]$ can be chosen as a set of three $\mathrm{C}^{\infty}$ functions $\varphi_{1}(x), \varphi_{2}(x), \varphi_{3}(x)$ where $\varphi_{1}$ is a cutoff function

$$
\varphi_{1}(x)=1 \quad \text { if } x \leq 1 / 4, \quad \varphi_{1}(x)=0 \quad \text { if } x \geq 1 / 2 .
$$

Similarily,

$$
\varphi_{3}(x)=0 \quad \text { if } x \leq 1 / 2, \quad \varphi_{3}(x)=1 \quad \text { if } x \geq 3 / 4,
$$

and

$$
\varphi_{2}(x)=1-\varphi_{1}(x)-\varphi_{3}(x)
$$

We now define the functions

$$
u_{i}(x, t)=\varphi_{i}(x) u(x, t), \quad i=1,2,3 .
$$

Clearly $u(x, t)=u_{1}(x, t)+u_{2}(x, t)+u_{3}(x, t)$ for all $x \in[0,1]$ and

$$
u_{i t t}=\varphi_{i} u_{t t}=\varphi_{i} u_{x x}=u_{i x x}+L_{i},
$$

where

$$
L_{i}=-2 \varphi_{i x}\left(u_{1 x}+u_{2 x}+u_{3 x}\right)-\varphi_{i x x}\left(u_{1}+u_{2}+u_{3}\right),
$$

consist only of lower order terms and has the same support as $u_{i}$. Defining also $f_{1 i}(x)=\varphi_{i}(x) f_{1}(x)$ and $f_{2 i}=\varphi_{i}(x) f_{2}(x)$ we obtain that $u_{1}$ solves the half line problem

$$
\begin{aligned}
u_{1 t t} & =u_{1 x x}+L_{1}, \quad x \in[0, \infty), \quad t \geq 0, \\
u_{1}(x, 0) & =f_{11}(x), \quad u_{1 t}(x, 0)=f_{21}(x), \quad B_{0} u_{1}(0, t)=g_{0}(t) .
\end{aligned}
$$

$u_{2}$ solves the initial value problem on the whole line

$$
u_{2 t t}=u_{2 x x}+L_{2}, \quad u_{2}(x, 0)=f_{12}(x), \quad u_{2 t}(x, 0)=f_{22}(x), \quad x \in(-\infty, \infty), \quad t \geq 0,
$$

and $u_{3}$ solves the half line problem

$$
\begin{aligned}
u_{3 t t} & =u_{3 x x}+L_{3}, \quad x \in(-\infty, 1], \quad t \geq 0, \\
u_{3}(x, 0) & =f_{13}(x), \quad u_{3 t}(x, 0)=f_{23}(x), \quad B_{1} u_{3}(1, t)=g_{1}(t) .
\end{aligned}
$$

If the three problems for $u_{1}, u_{2}$ and $u_{3}$ are well posed, then the original problem (2.10)-(2.11) is well posed.

To treat variable coefficient problems one can invoke what is known as the "principle of frozen coefficients" to replace the problem by one with constant coefficients. Heuristically, one can think that if one localizes the problem to very small regions then the coefficients of the equation in each region are nearly constant and the behavior of the solution is near to that of an equation with constant (frozen) coefficients. The proof of the validity of this requires the use of pseudo-differential theory. Here we claim the validity of this principle for our problem but do not go into the details.

\subsection{Stability against lower order perturbations}

If the problem is strongly boundary stable, then it is strongly stable and therefore stable against lower order perturbations. We shall now prove that the corresponding results hold for well posed problems. 
Theorem 2.9. Consider the problem (2.1)-(2.3) for $F=0, g \neq 0$ and change the boundary conditions to

$$
L u=g+l u, \quad|l| \text { bounded. }
$$

If the problem is boundary stable, then the same is true for the perturbed problem.

Proof. We consider $l u$ as part of the data. Then (2.5) becomes

$$
\int_{0}^{T} \mathrm{e}^{-2 \eta t}\|u(\cdot, t)\|_{\mathrm{H}^{0}(\Gamma)}^{2} \mathrm{~d} t \leq \frac{2}{\eta^{\alpha}}\left(|l|^{2} \int_{0}^{T} \mathrm{e}^{-2 \eta t}\|u(\cdot, t)\|_{\mathrm{H}^{0}(\Gamma)}^{2} \mathrm{~d} t+\int_{0}^{T} \mathrm{e}^{-2 \eta t}\|g(\cdot, t)\|_{\mathrm{H}^{0}(\Gamma)}^{2} \mathrm{~d} t\right) .
$$

By choosing $\eta_{0}$ such that $\frac{K}{\eta_{0}^{\alpha}}|l|^{2} \leq \frac{1}{4}$, we obtain an estimate also for the perturbed problem.

Theorem 2.10. Consider the problem (2.1)-(2.3) with $g=0$ and change the differential equations to

$$
u_{t t}=P_{0}(D) u+P_{1}(D) u+F .
$$

Here $P_{1}(D)$ is a first order differential operator with bounded coefficients, i.e.,

$$
\left\|P_{1}(D) u\right\|_{\mathrm{H}^{0}(\Omega)}^{2} \leq K_{1}\|u\|_{\mathrm{H}^{1}(\Omega)}^{2} .
$$

Assume that our problem is stable. Then the perturbed problem has the same property.

Proof. In the same way as in Theorem 2.9 we consider $P_{1}(D) u$ as part of the forcing and choose $\eta_{0}$ and $\alpha$ sufficiently large. Then the desired estimate follows.

Remark 2.11. One could argue that there are too many definitions. The reason for the concept of boundary stabillity is that it gives the easiest test for wellposedness. If the problem is strongly boundary stable then this test provides sufficient conditions for wellposedness. If the problem is only boundary stable we can use the test to find out what kind of boundary phenomena the problem has. This knowledge is crucial in constructing numerical methods. In the boundary stable case, the test provides only necessary conditions; to obtain wellposedness one needs to show that the estimate (2.7) also holds. This is always true if there is an energy estimate for homogeneous boundary data.

Remark 2.12. If we do not want to distinguish between strongly stable and stable, we call the problem well posed.

\section{A single Wave EQUATion}

\subsection{A necessary condition for well posedness}

To derive necessary conditions we consider in this section the halfplane problem for the wave equation

$$
u_{t t}=u_{x x}+u_{y y}+F(x, y, t), \quad x \geq 0,-\infty<y<\infty, t \geq 0
$$

with initial conditions, at $t=0$,

$$
u(x, y, 0)=f_{1}(x, y), \quad u_{t}(x, y, 0)=f_{2}(x, y)
$$

and one of four types of boundary conditions at $x=0,-\infty<y<\infty$ :

$$
\begin{aligned}
& \text { (1) } u_{t}=a u_{x}+b u_{y}+g, \quad a, b \text { real, }|b|<1, a>0 \\
& \text { (2) } u_{x}=i b u_{y}+g, \quad b \text { real, } b \neq 0,|b|<1 \\
& \text { (3) } u_{x}=g \\
& \text { (4) } u_{x}=b u_{y}+g, \quad b \text { real, } b \neq 0 .
\end{aligned}
$$


The source $F$ and the data $f_{j}, g$ are compatible smooth functions with compact support. We are only interested in solutions with bounded $\mathrm{L}^{2}$-norm and therefore we assume

$$
\int_{-\infty}^{\infty} \int_{0}^{\infty}|u(x, y, t)|^{2} \mathrm{~d} x \mathrm{~d} y=\|u\|^{2}<\infty \quad \text { for every fixed } t .
$$

We start with a test to find a necessary condition such that the problem is well posed. In this chapter and throughout the rest of the paper, $s=\eta+\mathrm{i} \xi$ denotes a complex number where $\eta, \xi \in \mathbb{R}$.

Lemma 3.1. Let $F=g=0$. The problem (3.1)-(3.2) is not well posed if we can find a nontrivial simple wave solution of type

$$
u=\mathrm{e}^{s t+\mathrm{i} \omega y} \varphi(x), \quad\|\varphi(x)\|<\infty, \quad \operatorname{Re} s>0 .
$$

Proof. If we have found such a solution, then

$$
u_{\alpha}=\mathrm{e}^{s \alpha t+\mathrm{i} \omega \alpha y} \varphi(\alpha x), \quad \alpha>0 .
$$

is also a solution for any $\alpha>0$. Since $\operatorname{Re} s>0$, we can find solutions that grow arbitrarily fast exponentially.

We shall now discuss whether there are such solutions. Introducing (3.4) into the homogeneous differential equation (3.1) and homogeneous boundary conditions (3.2) gives us

$$
\varphi_{x x}-\left(s^{2}+\omega^{2}\right) \varphi=0, \quad\|\varphi\|<\infty .
$$

(3.5) is an ordinary differential equation with constant coefficients and boundary conditions

$$
\begin{aligned}
& \text { (1) } s \varphi(0)=a \varphi_{x}(0)+b \mathrm{i} \omega \varphi(0), \quad a, b \text { real, }|b|<1, a>0, \\
& \text { (2) } \varphi_{x}(0)=-b \omega \varphi(0), \quad b \text { real, } b \neq 0,|b|<1, \\
& (3) \varphi_{x}(0)=0 \\
& \text { (4) } \varphi_{x}(0)=b \operatorname{i\omega } \varphi(0), \quad b \text { real, } b \neq 0 .
\end{aligned}
$$

The general solution of (3.5) is of the form

$$
\varphi(x)=\sigma_{1} \mathrm{e}^{\kappa x}+\sigma_{2} \mathrm{e}^{-\kappa x},
$$

where $\pm \kappa$ are the solutions of the characteristic equation

$$
\kappa^{2}-\left(s^{2}+\omega^{2}\right)=0, \quad \text { i.e. }, \quad \kappa=\sqrt{s^{2}+\omega^{2}} .
$$

We fix the argument of $\sqrt{ }$ by

$$
-\pi<\arg \left(s^{2}+\omega^{2}\right) \leq \pi, \quad \arg \sqrt{s^{2}+\omega^{2}}=\frac{1}{2} \arg \left(s^{2}+\omega^{2}\right) .
$$

From the general theory (also proved in Lemma A.5 in the appendix) we know that there is a constant $\delta>0$, such that

$$
\operatorname{Re} \kappa \geq \delta \operatorname{Re} s .
$$

Therefore $\varphi \in \mathrm{L}^{2}$ if and only if $\sigma_{1}=0$. Introducing (3.7) into the boundary conditions gives us

$$
\begin{aligned}
& \text { (1) } s=-a \kappa+\mathrm{i} \omega b, \quad a, b \text { real, }|b|<1, a>0, \\
& \text { (2) } \kappa=\omega b, \quad b \text { real, } b \neq 0,|b|<1, \\
& \text { (3) } \kappa=0, \\
& \text { (4) } \kappa=-\mathrm{i} \omega b, \quad b \text { real, } b \neq 0 .
\end{aligned}
$$


Since, by assumption, $a>0$ and $\operatorname{Re} \kappa>0$, there are no solutions of type (3.4) for the first kind of boundary condition. It is important to stress here that chosing the wrong sign for $a$ in the first type of boundary condition results into an ill posed problem and no solution can be computed. The second case in (3.8) implies

$$
\omega^{2}+s^{2}=\omega^{2} b^{2}, \quad \text { i.e., } \quad s^{2}=\omega^{2}\left(b^{2}-1\right) .
$$

Thus there are no solutions of type (3.4) for $|b|<1, b$ real. As Re $\kappa>0$ there are no solution of type (3.4) for the third boundary condition. Finally, since Re $\kappa>0$ and $b$ is real, there are no simple wave solutions of type (3.4) for the fourth kind of boundary condition.

We have proved

Theorem 3.2. For the boundary conditions (3.2) there are no simple wave solutions of type (3.4) other than the trivial solution $u \equiv 0$.

Since (3.5) and (3.8) define eigenvalue problems, we can phrase the theorem also as

Theorem 3.3. The eigenvalue problems (3.5) and (3.8) have no eigenvalues with $\operatorname{Re} s>0$.

We shall now introduce the concept of generalized eigenvalues. For that purpose we write (3.8) in terms of normalized variables.

$$
s^{\prime}=s / \sqrt{|s|^{2}+\omega^{2}}, \quad \omega^{\prime}=\omega / \sqrt{|s|^{2}+\omega^{2}}, \quad \kappa^{\prime}=\kappa / \sqrt{|s|^{2}+\omega^{2}} .
$$

Definition 3.4. Let $s^{\prime}=\mathrm{i} \xi_{0}^{\prime}, \omega^{\prime}=\omega_{0}^{\prime}$ be a fixed point and consider (3.5), (3.8) for $s^{\prime}=\mathrm{i} \xi_{0}^{\prime}+\eta^{\prime}, \omega^{\prime}=\omega_{0}^{\prime}, \eta^{\prime}>0$. $\left(\mathrm{i} \xi_{0}^{\prime}, \omega_{0}^{\prime}\right)$ is a generalized eigenvalue for a boundary condition if, in the limit $\eta^{\prime} \rightarrow 0$, the boundary condition is satisfied.

We now calculate the generalized eigenvalues. By Lemma A.7, there are no generalized eigenvalues for boundary conditions of type (1).

For boundary conditions of type (2), we need to consider

$$
\lim _{\eta^{\prime} \rightarrow 0}\left(\sqrt{\left(\mathrm{i} \xi_{0}^{\prime}+\eta^{\prime}\right)^{2}+\omega_{0}^{\prime 2}}-b \omega_{0}^{\prime}\right) .
$$

As $\operatorname{Re} \kappa^{\prime} \geq 0$, there will be a generalized eigenvalue for boundary condition (2) if and only if $b \omega_{0}^{\prime}>0$ and

$$
-\xi_{0}^{\prime 2}+\omega_{0}^{\prime 2}=b^{2} \omega_{0}^{\prime 2}, \quad \text { i.e., } \quad \xi_{0}^{\prime}= \pm \sqrt{1-b^{2}} \omega_{0}^{\prime} .
$$

Since

$$
\kappa_{0}^{\prime}=\sqrt{-\xi_{0}^{2}+\omega_{0}^{\prime 2}}=b \omega_{0}^{\prime}>0
$$

the corresponding eigenfunctions are

$$
u=\mathrm{e}^{-\left|b \omega_{0}\right| x} \mathrm{e}^{\mathrm{i} \omega_{0}\left(y \pm \sqrt{1-b^{2}} t\right)} .
$$

They represent surface waves that decay exponentially in $x$, i.e. in the normal direction away from the boundary. They are important phenomena in many applications (e.g. elastic wave equations).

For boundary conditions (3) and (4) $\xi_{0}^{\prime}, \omega_{0}^{\prime}$ must satisfy the relation

$$
\xi_{0}^{\prime}= \pm \sqrt{1+b^{2}} \omega_{0}^{\prime}, \quad \kappa_{0}^{\prime}=-\mathrm{i} \omega_{0}^{\prime} b,
$$

and where the sign in the first relation is chosen so that $\xi_{0}^{\prime} b \omega_{0}^{\prime}<0$ (because $R e \kappa^{\prime} \geq 0$.) The corresponding eigenfunction is

$$
u=\mathrm{e}^{\mathrm{i} \omega_{0}(b x+y)} \mathrm{e}^{ \pm \mathrm{i} \sqrt{1+b^{2}}\left|\omega_{0}\right| t} .
$$


For boundary condition $(4)(b \neq 0)$, they are oscillatory in $x, y, t$. For boundary condition $(3)(b=0)$, they are constant normal to the boundary and they are called glancing waves. They are important physical phenomena (e.g. Maxwell's equations).

We collect the results in

Theorem 3.5. There are no generalized eigenvalues for boundary conditions of type (1). For boundary condition (2), (3) and (4) the generalized eigenvalues are given by

$$
\begin{aligned}
& \text { (2) } \xi_{0}^{\prime}= \pm \sqrt{1-b^{2}} \omega_{0}^{\prime}, \quad \kappa_{0}^{\prime}=b \omega_{0}^{\prime}>0, \\
& \text { (3) }\left|\xi_{0}^{\prime}\right|=\left|\omega_{0}^{\prime}\right|, \quad \kappa_{0}^{\prime}=0, \\
& \text { (4) }\left|\xi_{0}^{\prime}\right|=\sqrt{1+b^{2}}\left|\omega_{0}^{\prime}\right|, \quad \kappa_{0}^{\prime}=-\mathrm{i} \omega_{0}^{\prime} b, \quad \xi_{0}^{\prime} b \omega_{0}^{\prime}<0 .
\end{aligned}
$$

\subsection{Reduction to a first order system of pseudo-differential equations}

The estimates obtained in this and subsequent sections are expressed in Fourier-Laplace transformed space. It is clear that all these estimates have their counterpart in physical space (such as the estimates in the definitions of Sect. 2.1). To understand the relation between both types of estimates we refer to Chapter 7.4 of [4] and Chapter 10 of [2].

We consider (3.1)-(3.3) with homogeneous initial data. We Laplace transform the problem with respect to $t$, Fourier transform it with respect to $y$, and denote the dual variables by $s, \omega$, respectively. For $\operatorname{Re} s>0$ we obtain

$$
\hat{u}_{x x}=\left(s^{2}+\omega^{2}\right) \hat{u}+\hat{F}, \quad \hat{u}=\hat{u}(x, \omega, s), \hat{F}=\hat{F}(x, \omega, s), 0 \leq x<\infty,
$$

with one of the boundary conditions

$$
\begin{aligned}
& \text { (1) } s \hat{u}=a \hat{u}_{x}+\mathrm{i} b \omega \hat{u}+\hat{g} \\
& \text { (2) } \hat{u}_{x}=-b \omega \hat{u}+\hat{g} \\
& \text { (3) } \hat{u}_{x}=\hat{g} \\
& \text { (4) } \hat{u}_{x}=\mathrm{i} b \omega \hat{u}+\hat{g}
\end{aligned}
$$

and $\|\hat{u}(\cdot, \omega, s)\|^{2}<\infty$.

Introducing a new variable by

$$
\hat{u}_{x}=\sqrt{|s|^{2}+\omega^{2}} \hat{v}
$$

we write the Fourier and Laplace transformed system as a first order system

$$
\hat{\mathbf{u}}_{x}=\sqrt{|s|^{2}+\omega^{2}} M \hat{\mathbf{u}}+\hat{\mathbf{F}}_{0} .
$$

Here

with

$$
\hat{\mathbf{u}}=\left(\begin{array}{c}
\hat{u} \\
\hat{v}
\end{array}\right), \quad M=\left(\begin{array}{cc}
0 & 1 \\
\kappa^{\prime 2} & 0
\end{array}\right), \quad \hat{\mathbf{F}}_{0}=\frac{1}{\sqrt{|s|^{2}+\omega^{2}}}\left(\begin{array}{c}
0 \\
\hat{F}
\end{array}\right)
$$

$$
\kappa^{\prime}=\sqrt{\left(s^{\prime}\right)^{2}+\left(\omega^{\prime}\right)^{2}}, \quad s^{\prime}=\frac{s}{\sqrt{|s|^{2}+\omega^{2}}}, \quad \omega^{\prime}=\frac{\omega}{\sqrt{|s|^{2}+\omega^{2}}} .
$$

The eigenvalues $\mu$ of $M$ are $\mu_{1}=-\kappa^{\prime}$ and $\mu_{2}=\kappa^{\prime}$. The boundary conditions at $x=0$ become

$$
\begin{aligned}
& \text { (1) } s^{\prime} \hat{u}=a \hat{v}+\mathrm{i} b \omega^{\prime} \hat{u}+g^{\prime}, \quad a>0, \quad|b|<1, \\
& \text { (2) } \hat{v}=-b \omega^{\prime} \hat{u}+g^{\prime}, \quad|b|<1, \quad b \neq 0, \\
& \text { (3) } \hat{v}=g^{\prime}, \\
& \text { (4) } \hat{v}=\mathrm{i} b \omega^{\prime} \hat{u}+g^{\prime}, \quad b \text { real, } b \neq 0,
\end{aligned}
$$

with $g^{\prime}=\hat{g} / \sqrt{|s|^{2}+\omega^{2}}$. 
Remark 3.6. We present in this and the following section the easiest way to obtain the estimates at the boundary and in the interior of the domain. To generalize these results to variable coefficients pseudo-differential theory is needed. The transformations $S$ and $T$ introduced below (see Eqs. (3.16), (3.31)) need to be smooth in the dual variables. The smoothness condition may fail only at the double root of $M$. In this case the Kreiss' symmetrizer is used to get the estimates as explained in [3].

We shall now calculate the solution for the case when $F=0$, and estimate it on the boundary.

The eigenvector of $M$ connected with $-\kappa^{\prime}$ is given by

$$
\mathbf{x}=\left(\begin{array}{c}
1 \\
-\kappa^{\prime}
\end{array}\right)
$$

The transformation

$$
S=\left(\begin{array}{cc}
1 & \bar{\kappa}^{\prime} \\
-\kappa^{\prime} & 1
\end{array}\right)
$$

is, except for a trivial normalization, unitary and transforms $M$ into upper triangular form, i.e.,

$$
S^{-1} M S=\left(\begin{array}{cc}
-\kappa^{\prime} & d \\
0 & \kappa^{\prime}
\end{array}\right), \quad d=\frac{1+\left|\kappa^{\prime}\right|^{4}}{1+\left|\kappa^{\prime}\right|^{2}} .
$$

Here $S, S^{-1}, d$ are uniformly bounded and depend smoothly on $\kappa^{\prime}$.

Introducing a new variable by

$$
\left(\begin{array}{l}
\hat{u} \\
\hat{v}
\end{array}\right)=S\left(\begin{array}{l}
\tilde{u} \\
\tilde{v}
\end{array}\right)
$$

transforms (3.13) into

$$
\left(\begin{array}{l}
\tilde{u} \\
\tilde{v}
\end{array}\right)_{x}=\left(\begin{array}{cc}
-\kappa d \sqrt{|s|^{2}+\omega^{2}} \\
0 & \kappa
\end{array}\right)\left(\begin{array}{l}
\tilde{u} \\
\tilde{v}
\end{array}\right) .
$$

As the solution is in $\mathrm{L}^{2}$, we have $\tilde{v}=0$ and also $\hat{u}=\tilde{u}$. Thus the boundary conditions become

$$
\begin{aligned}
& \text { (1) }\left(s^{\prime}+a \kappa^{\prime}-\mathrm{i} b \omega^{\prime}\right) \tilde{u}=g^{\prime}, \\
& \text { (2) }\left(\kappa^{\prime}-b \omega^{\prime}\right) \tilde{u}=-g^{\prime}, \\
& \text { (3) } \kappa^{\prime} \tilde{u}=-g^{\prime}, \\
& \text { (4) }\left(\kappa^{\prime}+\mathrm{i} b \omega^{\prime}\right) \tilde{u}=-g^{\prime} .
\end{aligned}
$$

By (3.8), these boundary conditions become singular exactly at the generalized eigenvalues.

Remark 3.7. From all the boundary conditons the first one is the most benign. By Lemma A.7 we obtain the estimate on the boundary

$$
|\tilde{u}(0, \omega, s)|^{2} \leq \frac{\text { const. }}{|s|^{2}+\omega^{2}}|\hat{g}(\omega, s)|^{2} .
$$

In this case we gain a derivative on the boundary and the problem is strongly boundary stable. According to the classical theory $[3,4]$ the problem is strongly stable. Moreover, the principle of localization holds and the problem can be generalized to variable coefficients and then to quasilinear equations. It is worth noticing here that away from generalized eigenvalues, i.e. when the factor in front of $\tilde{u}$ on the left hand side of (3.20) are strictly away from zero, the estimate (3.21) holds also for boundary conditions (2), (3) and (4) and therefore the problem can be treated by the classical theory.

Because of the previous remark, we only need to study the estimates near the generalized eigenvalues. We have 
Theorem 3.8. The problem (3.1)-(3.3) with $F=0$ and $f_{1}=f_{2}=0$ has a unique solution in $\mathrm{L}^{2}$ which, Fourier-Laplace transformed, is given by

$$
\hat{u}=\mathrm{e}^{-\kappa x} \hat{u}(0, \omega, s), \quad \kappa=\sqrt{s^{2}+\omega^{2}} .
$$

For the different boundary conditions sharp estimates follow. For boundary condition (1) and, "away" from generalized eigenvalues, for all other boundary conditions the problem is strongly boundary stable and

$$
\text { (1) }|\hat{u}(0, \omega, s)|^{2} \leq \frac{\text { const. }}{|s|^{2}+\omega^{2}}|\hat{g}|^{2} .
$$

Near generalized eigenvalues the estimates for boundary conditions (2), (3) and (4) are

$$
\begin{aligned}
& \text { (2) }|\hat{u}(0, \omega, s)|^{2} \leq \frac{\text { const. }}{\eta^{2}}|\hat{g}|^{2} \\
& \text { (3) }|\hat{u}(0, \omega, s)|^{2} \leq \text { const. } \frac{|\hat{g}|^{2}}{|\kappa|^{2}} \leq \text { const. } \frac{|\hat{g}|^{2}}{\eta\left(|s|^{2}+\omega^{2}\right)^{1 / 2}}, \\
& \text { (4) }|\hat{u}(0, \omega, s)|^{2} \leq \frac{\text { const. }}{\eta^{2}}|\hat{g}|^{2}
\end{aligned}
$$

and the problem is boundary stable.

Proof. Clearly, as $\tilde{v}=0$ and $\hat{u}=\tilde{u},(3.22)$ is the only solution to (3.19). We need to consider only a neighbourhood of the generalized eigenvalues $\left(\mathrm{i} \xi_{0}^{\prime}, \omega_{0}^{\prime}\right)$. For the second boundary condition $\xi_{0}^{\prime}= \pm \sqrt{1-b^{2}} \omega_{0}^{\prime}$, i.e.,

$$
s^{\prime}=i\left(\xi_{0}^{\prime}+\tilde{\xi}^{\prime}\right)+\eta^{\prime}, \quad \eta^{\prime} \geq 0, \quad \omega^{\prime}=\omega_{0}^{\prime}+\tilde{\omega}^{\prime}, \quad\left|\tilde{\xi}^{\prime}\right|+\left|\tilde{\omega}^{\prime}\right|+\eta^{\prime} \ll 1 .
$$

Since $\kappa^{\prime} \neq 0$ at $\left(\mathrm{i} \xi_{0}^{\prime}, \omega_{0}^{\prime}\right)$, we can use Taylor expansion. A simple perturbation caculation shows that the worst estimate occurs for $\tilde{\xi}^{\prime}=\tilde{\omega}^{\prime}=0$. In this case we have,

$$
\begin{aligned}
\left|\kappa^{\prime}-b \omega^{\prime}\right| & =\left|\sqrt{-\xi_{0}^{\prime 2}+2 \mathrm{i} \xi_{0}^{\prime} \eta^{\prime}+\eta^{\prime 2}+\omega_{0}^{\prime 2}}-b \omega_{0}^{\prime}\right| \\
& =\left|\sqrt{b^{2} \omega_{0}^{\prime 2}+2 \mathrm{i} \xi_{0}^{\prime} \eta^{\prime}+\eta^{\prime 2}}-b \omega_{0}^{\prime}\right| \\
& \approx|| b \omega_{0}^{\prime}\left|-b \omega_{0}^{\prime}+\frac{\mathrm{i} \xi_{0}^{\prime} \eta^{\prime}}{\left|b \omega_{0}^{\prime}\right|}\right| \approx \frac{\sqrt{1-b^{2}}}{|b|} \eta^{\prime} \quad \text { if } \quad b \omega_{0}^{\prime}>0 .
\end{aligned}
$$

Thus we have

$$
|\tilde{u}(0, \omega, s)|^{2} \leq \mathrm{const} \cdot \frac{\left|g^{\prime}\right|^{2}}{\eta^{\prime 2}}=\text { const. } \frac{|\hat{g}|^{2}}{\eta^{2}} .
$$

A similar perturbation calculation gives

$$
\left|\kappa^{\prime}+\mathrm{i} b \omega^{\prime}\right| \simeq \frac{\sqrt{1+b^{2}}}{|b|} \eta^{\prime}+\mathcal{O}\left(\eta^{\prime 2}\right)
$$

which gives, for the last boundary condition

$$
|\tilde{u}(0, \omega, s)|^{2} \leq \text { const. } \frac{|\hat{g}|^{2}}{\eta^{2}} .
$$

For the third boundary condition one can do better. Lemma A. 4 with $b=0$, gives us

$$
|\tilde{u}(0, \omega, s)|^{2} \leq \text { const. }\left(\frac{\left|g^{\prime}\right|}{\left|\kappa^{\prime}\right|}\right)^{2}=\text { const. }\left(\frac{|\hat{g}|}{|\kappa|}\right)^{2} \leq \frac{\text { const. }}{\eta} \frac{|\hat{g}|^{2}}{\left(|s|^{2}+\omega^{2}\right)^{1 / 2}} .
$$

This proves the theorem. 
Theorem 3.9. When $f_{1}=f_{2}=0$ and $F=0$, the unique solution to our problem, described in Theorem 3.8, satisfies the following interior estimates. For boundary condition (1) and, away from generalized eigenvalues, for boundary conditions (2), (3) and (4)

$$
\|\tilde{u}\|^{2} \leq \frac{\text { const. }}{\eta} \frac{|\hat{g}|^{2}}{|s|^{2}+\omega^{2}}
$$

Close to generalized eigenvalues, for the corresponding boundary conditions, we have

$$
\begin{aligned}
& \text { (2) }\|\tilde{u}\|^{2} \leq \frac{\text { const. }}{\eta^{2}} \frac{|\hat{g}|^{2}}{\left(|s|^{2}+\omega^{2}\right)^{1 / 2}}, \\
& \text { (3) }\|\tilde{u}\|^{2} \leq \frac{\text { const. }}{\eta^{3 / 2}} \frac{|\hat{g}|^{2}}{\left(|s|^{2}+\omega^{2}\right)^{3 / 4}}, \\
& \text { (4) }\|\tilde{u}\|^{2} \leq \frac{\text { const. }}{\eta^{3}}|\hat{g}|^{2} .
\end{aligned}
$$

Proof. By Theorem 3.8 and Lemma A.2, the solution satifies

$$
\|\tilde{u}\|^{2} \leq \frac{1}{\operatorname{Re} \kappa}|\tilde{u}(0, \omega, s)|^{2} .
$$

Since, by Lemma A.5, always Re $\kappa \geq \delta_{4} \eta$, we obtain from (3.23) that (3.28) is valid. (2) follows because, by Theorem 3.5, Re $\kappa^{\prime} \simeq b \omega_{0}^{\prime}$ and then Re $\kappa \geq$ const. $\sqrt{|s|^{2}+\omega^{2}}$. (3) follows from (3.27), Lemma A.6 and Lemma A.4, according to

$$
\|\tilde{u}\|^{2} \leq \text { const. } \frac{|\hat{g}|^{2}}{\operatorname{Re} \kappa|\kappa|^{2}} \leq \text { const. } \frac{|\hat{g}|^{2}}{\eta|\kappa| \sqrt{|s|^{2}+\omega^{2}}} \leq \text { const. } \frac{|\hat{g}|^{2}}{\eta^{3 / 2}\left(|s|^{2}+\omega^{2}\right)^{3 / 4}}
$$

Finally (4) corresponds to (4) of (3.24). This proves the theorem.

\subsection{Estimates for homogeneous boundary data}

We consider now the problem (3.13), (3.14) with $g^{\prime}=0$ and treat only the cases (2), (3) and (4) where there are generalized eigenvalues (see Rem. 3.7).

For $\eta=\operatorname{Re} s>0$, the eigenvalues of $M$ are distinct and therefore we can transform (3.13) to diagonal form by the transformation

$$
T=\left(\begin{array}{cc}
1 & 1 \\
-\kappa^{\prime} & \kappa^{\prime}
\end{array}\right), \quad T^{-1}=\frac{1}{2}\left(\begin{array}{cc}
1 & -1 / \kappa^{\prime} \\
1 & 1 / \kappa^{\prime}
\end{array}\right)
$$

Let $\tilde{u}, \tilde{v}$ be defined by

$$
\left(\begin{array}{l}
\hat{u} \\
\hat{v}
\end{array}\right)=T\left(\begin{array}{l}
\tilde{u} \\
\tilde{v}
\end{array}\right)
$$

Then, (3.13) becomes

$$
\left(\begin{array}{l}
\tilde{u} \\
\tilde{v}
\end{array}\right)_{x}=\left(\begin{array}{cc}
-\kappa & 0 \\
0 & \kappa
\end{array}\right)\left(\begin{array}{l}
\tilde{u} \\
\tilde{v}
\end{array}\right)+\frac{1}{2 \kappa^{\prime} \sqrt{|s|^{2}+\omega^{2}}}\left(\begin{array}{c}
-\hat{F} \\
\hat{F}
\end{array}\right),
$$

with boundary conditions

(2) $\left(\kappa^{\prime}-b \omega^{\prime}\right) \tilde{u}(0, \omega, s)=\left(\kappa^{\prime}+b \omega^{\prime}\right) \tilde{v}(0, \omega, s)$,

(3) $\tilde{u}(0, \omega, s)=\tilde{v}(0, \omega, s)$,

(4) $\left(\kappa^{\prime}+\mathrm{i} b \omega^{\prime}\right) \tilde{u}(0, \omega, s)=\left(\kappa^{\prime}-\mathrm{i} b \omega^{\prime}\right) \tilde{v}(0, \omega, s)$. 
Equations (3.33) are decoupled and as $\operatorname{Re} \kappa>0$, Lemma A.1 gives for all boundary conditions

$$
\begin{aligned}
& |\tilde{v}(0, \omega, s)|^{2} \leq \frac{1}{8\left|\kappa^{\prime}\right|^{2} \operatorname{Re} \kappa} \frac{\|\hat{F}\|^{2}}{|s|^{2}+\omega^{2}}=\frac{1}{8\left|\kappa^{\prime}\right|^{2} \operatorname{Re} \kappa^{\prime}} \frac{\|\hat{F}\|^{2}}{\left(|s|^{2}+\omega^{2}\right)^{3 / 2}}, \\
& \|\tilde{v}(\cdot, \omega, s)\|^{2} \leq \frac{1}{4\left|\kappa^{\prime}\right|^{2}|\operatorname{Re} \kappa|^{2}} \frac{\|\hat{F}\|^{2} \mid}{|s|^{2}+\omega^{2}}=\frac{1}{4\left|\kappa^{\prime}\right|^{2}\left|\operatorname{Re} \kappa^{\prime}\right|^{2}} \frac{\|\hat{F}\|^{2}}{\left(|s|^{2}+\omega^{2}\right)^{2}} .
\end{aligned}
$$

We use the boundary conditions to estimate $\tilde{u}(0, \omega, s)$.

Theorem 3.5 tells us that $\operatorname{Re} \kappa_{0}^{\prime} \approx b \omega_{0}^{\prime}>0$ in a neighborhood of the generalized eigenvalue connected with boundary condition (2). Therefore the perturbation calculation (3.25) gives us

$$
\begin{aligned}
|\tilde{u}(0, \omega, s)|^{2} & =\left|\frac{\kappa^{\prime}+b \omega^{\prime}}{\kappa^{\prime}-b \omega^{\prime}}\right|^{2}|\tilde{v}(0, \omega, s)|^{2} \leq \frac{\text { const. }}{\eta^{\prime 2}} \frac{\|\hat{F}\|^{2}}{\left(|s|^{2}+\omega^{2}\right)^{3 / 2}} \\
& \leq \frac{\text { const. }}{\eta^{2}} \frac{\|\hat{F}\|^{2}}{\left(|s|^{2}+\omega^{2}\right)^{1 / 2}} .
\end{aligned}
$$

The interior estimate in this case follows from Lemma A.2, $\operatorname{Re} \kappa=\operatorname{Re} \kappa^{\prime} \sqrt{|s|^{2}+\omega^{2}}$ and (3.36)

$$
\begin{aligned}
\|\tilde{u}(\cdot, \omega, s)\|^{2} & \leq \frac{|\tilde{u}(0, \omega, s)|^{2}}{\operatorname{Re} \kappa}+\frac{\text { const. }}{(\operatorname{Re} \kappa)^{2}} \frac{\|\hat{F}\|^{2}}{|s|^{2}+\omega^{2}} \\
& \leq \frac{\text { const. }}{\eta^{2}} \frac{\|\hat{F}\|^{2}}{|s|^{2}+\omega^{2}} .
\end{aligned}
$$

Since the transformation $T$ is bounded, (3.32) tells us that the estimates (3.35)-(3.37) are also valid for $\hat{u}, \hat{v}$. Thus the problem is stable.

For boundary condition (3), the generalized eigenvalue is $\kappa^{\prime}=0$ and therefore, for $\operatorname{Re} s^{\prime}>0$, we know only that $\operatorname{Re} \kappa^{\prime} \geq \delta \operatorname{Re} s^{\prime}$. However, by Lemma A.6, we have a strong estimate for $|\kappa| \operatorname{Re} \kappa$ and the estimates (3.35) become

$$
\begin{aligned}
|\tilde{v}(0, \omega, s)|^{2} & \leq \frac{\text { const. }}{\eta|\kappa|} \frac{\|\hat{F}\|^{2}}{\sqrt{|s|^{2}+\omega^{2}}} \leq \frac{\text { const. }}{\eta^{2}} \frac{\|\hat{F}\|^{2}}{\sqrt{|s|^{2}+\omega^{2}}} \\
\|\tilde{v}(\cdot, \omega, s)\|^{2} & \leq \frac{\text { const. }}{\eta^{2}} \frac{\|\hat{F}\|^{2}}{|s|^{2}+\omega^{2}} .
\end{aligned}
$$

By (3.34), the same estimate holds for $\tilde{u}(0, \omega, s)$. By Lemma A.2, we obtain the interior estimate

$$
\|\tilde{u}(\cdot, \omega, s)\|^{2} \leq \frac{\text { const. }}{\eta^{2}} \frac{\|\hat{F}\|^{2}}{|s|^{2}+\omega^{2}} .
$$

Since $\hat{u}, \hat{v}$ satisfy the same estimates, the problem is stable.

For boundary condition (4), the generalized eigenvalue is $\kappa_{0}^{\prime}=-i \omega_{0}^{\prime} b, b \neq 0$, is purely imaginary. Therefore, for $\operatorname{Re} s^{\prime}>0$, we can only use the estimate $\operatorname{Re} \kappa^{\prime} \geq \delta \eta^{\prime}$. Instead of (3.36) we obtain now

$$
|\tilde{u}(0, \omega, s)|^{2}=\left|\frac{\kappa^{\prime}-\mathrm{i} \omega^{\prime} b}{\kappa^{\prime}+\mathrm{i} \omega^{\prime} b}\right|^{2}|\tilde{v}(0, \omega, s)|^{2} \leq \frac{\text { const. }}{\eta^{3}}\|\hat{F}\|^{2} .
$$

Again, the same estimates hold for $\hat{u}, \hat{v}$. The estimates are sharp. Therefore we do not obtain the desired interior estimate and the problem is unstable. We have proved

Theorem 3.10. For boundary conditions (2) and (3) our problem is stable, but not for boundary condition (4). 
Remark 3.11. The estimates obtained for boundary conditions (2) and (3) tell us that $\hat{u}$ gains one derivative with respect to the forcing in the interior of the domain and half a derivative on the boundary. The problem can be localized and generalized to variable coefficients and then to quasilinear equations. On the other hand, the estimates for the problem with boundary condition (4) show that not even a fractional derivative is gained with respect to the forcing. This, for a second order equation, means that one derivative of the solution is lost at every reflection on the boundary. The problem can not be localized. We do not pursue this problem but illustrate below this bad type of behavior with a simple example: a first order system with a boundary condition equivalent to (4).

An example: Boundary reflection with loss or gain of differentiability. Consider a system of differential equations

$$
u_{t}=-u_{x}, \quad v_{t}=v_{x}, \quad 0 \leq x \leq 1, \quad t>0,
$$

with boundary conditions

$$
u(0, t)=v_{x}(0, t), \quad v(1, t)=u_{x}(1, t) .
$$

Then

$$
u=\mathrm{e}^{\lambda(t-x)} u_{0}, \quad v=\mathrm{e}^{\lambda(t+x)} v_{0},
$$

is a solution of (3.40).

Introducing (3.42) into (3.41) gives us

$$
u_{0}=\lambda v_{0}, \quad \mathrm{e}^{\lambda} v_{0}=-\lambda \mathrm{e}^{-\lambda} u_{0} .
$$

Thus we obtain a solution of (3.40), (3.41) if

$$
\mathrm{e}^{2 \lambda}=-\lambda^{2} .
$$

Let

then (3.44) becomes

$$
\lambda=\lambda_{n}=\pi \mathrm{i} n+\tilde{\lambda}_{n}, \quad n=1,2, \ldots,
$$

(3.45) has a solution

$$
\mathrm{e}^{2 \tilde{\lambda}_{n}}=\pi^{2} n^{2}-2 \pi \mathrm{i} n \tilde{\lambda}_{n}-\tilde{\lambda}_{n}^{2} .
$$

$$
\tilde{\lambda}_{n} \approx \log \pi n
$$

Therefore the solution (3.42) grows like

$$
\mathrm{e}^{\lambda t} \simeq \mathrm{e}^{\pi \mathrm{i} n t} \cdot \mathrm{e}^{t \log \pi n}=\mathrm{e}^{\pi \mathrm{i} n t}(\pi n)^{t} .
$$

If the initial data can be expanded into a Fourier series

$$
u(x, 0)=\sum_{n} \mathrm{e}^{\lambda_{n} x} \hat{u}\left(\lambda_{n}\right),
$$

then (3.46) tells us that the solution loses more and more derivatives with time.

Now change the boundary conditions (3.41) to

$$
u_{x}(0, t)=v(0, t), \quad v_{x}(1, t)=u(1, t) .
$$

Then we obtain, instead of (3.44),

$$
\begin{gathered}
-\lambda u_{0}=v_{0}, \quad \lambda \mathrm{e}^{\lambda} v_{0}=\mathrm{e}^{-\lambda} u_{0}, \\
\mathrm{e}^{2 \lambda}=-\frac{1}{\lambda^{2}} .
\end{gathered}
$$

Therefore there is no loss of derivatives.

Geometrically, the two sets of boundary conditions represents two different situations. In the first case any wave loses a derivative when reflected at the boundary. In the second case, it gains a derivative. 


\section{SECOND ORDER SySTEMS OF HYPERBoliC EQUATIONS}

\subsection{Two wave equations}

In this section we consider two wave equations coupled through the boundary conditions.

$$
u_{1 t t}=u_{1 x x}+u_{1 y y}, \quad u_{2 t t}=u_{2 x x}+u_{2 y y},
$$

on the halfplane $x \geq 0,-\infty<y<\infty$, for $t \geq 0$, with homogeneous initial conditions

$$
u_{i}(x, y, 0)=u_{i t}(x, y, 0)=0, \quad i=1,2, \quad t=0,
$$

and boundary conditions at $x=0$,

$$
u_{1 x}+b_{1} u_{2 y}=g_{1}, \quad u_{2 x}+b_{2} u_{1 y}=g_{2}, \quad x=0 .
$$

Here $b_{1}, b_{2}$, are real and $g_{j}=g_{j}(y, t), j=1,2$, are smooth functions that are compatible with the initial data (for example, functions that vanish near $t=0$ ). We want to show that our techniques of Section 3 can still be used to describe the behavior of the solution.

Fourier and Laplace transformations lead to

$$
\hat{u}_{1 x x}-\left(\omega^{2}+s^{2}\right) \hat{u}_{1}=0, \quad \hat{u}_{2 x x}-\left(\omega^{2}+s^{2}\right) \hat{u}_{2}=0, \quad \operatorname{Re} s>0 .
$$

Thus we obtain solutions that belong to $\mathrm{L}^{2}$

$$
\hat{u}_{1}=\mathrm{e}^{s t+\mathrm{i} \omega y-\kappa x} u_{10}, \quad \hat{u}_{2}=\mathrm{e}^{s t+\mathrm{i} \omega y-\kappa x} u_{20},
$$

where

$$
\kappa=\sqrt{\omega^{2}+s^{2}}, \quad \text { for } \operatorname{Re} s>0 .
$$

We recall here that $\operatorname{Re} \kappa>0$ when $\operatorname{Re} s>0$. The transformed boundary conditions become

$$
\begin{aligned}
-\kappa u_{10}+\mathrm{i} \omega b_{1} u_{20} & =\hat{g}_{1}, \\
\mathrm{i} \omega b_{2} u_{10}-\kappa u_{20} & =\hat{g}_{2} .
\end{aligned}
$$

A simple calculation shows that (4.6) has a unique solution

$$
u_{10}=-\frac{\kappa \hat{g}_{1}+\mathrm{i} \omega b_{1} \hat{g}_{2}}{\kappa^{2}+\omega^{2} b_{1} b_{2}}, \quad u_{20}=-\frac{\kappa \hat{g}_{2}+\mathrm{i} \omega b_{2} \hat{g}_{1}}{\kappa^{2}+\omega^{2} b_{1} b_{2}}
$$

if and only if

$$
\operatorname{Det}\left(\begin{array}{cc}
-\kappa & \mathrm{i} \omega b_{1} \\
\mathrm{i} \omega b_{2} & -\kappa
\end{array}\right)=\kappa^{2}+\omega^{2} b_{1} b_{2} \neq 0 .
$$

There is an eigenvalue to the homogeneous problem (4.4), (4.6) with $\hat{g}_{1}=\hat{g}_{2}=0$, if the homogeneous system (4.6) has a nontrivial solution. By (4.6), this is the case if

$$
\kappa^{2}+\omega^{2} b_{1} b_{2}=s^{2}+\omega^{2}\left(b_{1} b_{2}+1\right)=0 .
$$

There are five different situations:

(1) $b_{1} b_{2}<-1$. By (4.9), there are eigenvalues $s$ with Re $s>0$. Therefore our problem is not well posed.

(2) $b_{1} b_{2}=-1$. Now $s=0$ is a generalized eigenvalue. For $s \neq 0$ the solution (4.7) becomes

$$
u_{10}=-\frac{\kappa \hat{g}_{1}+\mathrm{i} \omega b_{1} \hat{g}_{2}}{s^{2}}, \quad u_{20}=-\frac{\kappa \hat{g}_{2}+\mathrm{i} \omega b_{2} \hat{g}_{1}}{s^{2}} .
$$

In general, we can not expect that $\hat{u}_{10}, \hat{u}_{20}$ stay bounded for $s \rightarrow 0$. We need to assume that $g_{j}=\tilde{g}_{j t t}$ are the second time derivatives of smooth functions. 
(3) $-1<b_{1} b_{2}<0$. By (4.5), (4.9), we obtain generalized eigenvalues and eigenfunctions if $s= \pm \mathrm{i} \sqrt{1+b_{1} b_{2}}|\omega|$, $\kappa=\sqrt{\left|b_{1} b_{2}\right|} \omega$. The solution (4.7) is singular at the eigenvalues and $\hat{u}_{10}, \hat{u}_{20}$ have a first order pole. In physical space we obtain surface waves whose amplitudes become large for $b_{1} b_{2} \rightarrow-1$.

(4) $b_{1} b_{2}=0$. Now $s= \pm \omega, \kappa=0$ determine the generalized eigenvalues and eigenfunctions. The behavior is the same as for the Neuman problem. We obtain glancing waves.

(5) $b_{1} b_{2}>0$. In this case the generalized eigenvalues and eigenfunctions are determined by $s= \pm \mathrm{i} \sqrt{1+b_{1} b_{2}} \omega$, $\kappa= \pm \mathrm{i} \sqrt{b_{1} b_{2}} \omega$. The eigenfunctions are oscillatory and the solution behaves like the solution of the wave equation in Section 3.3 with boundary condition (4). Thus it is unstable.

In summary we can state that the problem (4.1)-(4.3) is well posed for boundary conditions (2)-(4) and unstable for boundary condition (5). Also, there can be numerical diffculties if $b_{1} b_{2}+1$ is zero or close to zero.

When we study the estimates for the problem in the cases (3) and (4) we get completely analogous estimates as the ones found for a single wave equation.

Consider the system

$$
u_{1 t t}=u_{1 x x}+u_{1 y y}-F_{1}, \quad u_{2 t t}=u_{2 x x}+u_{2 y y}-F_{2},
$$

with boundary conditions

$$
u_{1 x}+b_{1} u_{2 y}=0, \quad u_{2 x}+b_{2} u_{1 y}=0, \quad x=0 .
$$

Fourier and Laplace transformations give us

$$
\hat{u}_{1 x x}=\left(s^{2}+\omega^{2}\right) \hat{u}_{1}+\hat{F}_{1}, \quad \hat{u}_{2 x x}=\left(s^{2}+\omega^{2}\right) \hat{u}_{2}+\hat{F}_{2},
$$

and define

$$
\hat{u}_{1 x}=\sqrt{|s|^{2}+\omega^{2}} \hat{v}_{1}, \quad \hat{u}_{2 x}=\sqrt{|s|^{2}+\omega^{2}} \hat{v}_{2} .
$$

Thus, the first order form of the problem becomes

$$
\begin{aligned}
& \left(\begin{array}{l}
\hat{u}_{1} \\
\hat{v}_{1}
\end{array}\right)_{x}=\sqrt{|s|^{2}+\omega^{2}}\left(\begin{array}{cc}
0 & 1 \\
\kappa^{\prime 2} & 0
\end{array}\right)\left(\begin{array}{l}
\hat{u}_{1} \\
\hat{v}_{1}
\end{array}\right)+\frac{1}{\sqrt{|s|^{2}+\omega^{2}}}\left(\begin{array}{c}
0 \\
\hat{F}_{1}
\end{array}\right) \\
& \left(\begin{array}{l}
\hat{u}_{2} \\
\hat{v}_{2}
\end{array}\right)_{x}=\sqrt{|s|^{2}+\omega^{2}}\left(\begin{array}{cc}
0 & 1 \\
\kappa^{\prime 2} & 0
\end{array}\right)\left(\begin{array}{l}
\hat{u}_{2} \\
\hat{v}_{2}
\end{array}\right)+\frac{1}{\sqrt{|s|^{2}+\omega^{2}}}\left(\begin{array}{c}
0 \\
\hat{F}_{2}
\end{array}\right)
\end{aligned}
$$

with boundary conditions

$$
\begin{aligned}
& \sqrt{|s|^{2}+\omega^{2}} \hat{v}_{10}+\mathrm{i} \omega b_{1} \hat{u}_{20}=0, \\
& \sqrt{|s|^{2}+\omega^{2}} \hat{v}_{20}+\mathrm{i} \omega b_{2} \hat{u}_{10}=0 .
\end{aligned}
$$

We transform (4.13) to diagonal form. Let

$$
\left(\begin{array}{l}
\hat{u}_{j} \\
\hat{v}_{j}
\end{array}\right)=\left(\begin{array}{cc}
1 & 1 \\
-\kappa^{\prime} & \kappa^{\prime}
\end{array}\right)\left(\begin{array}{l}
\tilde{u}_{j} \\
\tilde{v}_{j}
\end{array}\right), \quad j=1,2 .
$$

Then

$$
\begin{aligned}
& \left(\begin{array}{l}
\tilde{u}_{1} \\
\tilde{v}_{1}
\end{array}\right)_{x}=\left(\begin{array}{cc}
-\kappa & 0 \\
0 & \kappa
\end{array}\right)\left(\begin{array}{l}
\tilde{u}_{1} \\
\tilde{v}_{1}
\end{array}\right)_{x}+\frac{1}{\sqrt{|s|^{2}+\omega^{2}}} \tilde{F}_{1} \\
& \left(\begin{array}{l}
\tilde{u}_{2} \\
\tilde{v}_{2}
\end{array}\right)_{x}=\left(\begin{array}{cc}
-\kappa & 0 \\
0 & \kappa
\end{array}\right)\left(\begin{array}{l}
\tilde{u}_{2} \\
\tilde{v}_{2}
\end{array}\right)_{x}+\frac{1}{\sqrt{|s|^{2}+\omega^{2}}} \tilde{F}_{2}
\end{aligned}
$$

with boundary conditions

$$
\begin{aligned}
-\kappa \tilde{u}_{10}+\mathrm{i} \omega b_{1} \tilde{u}_{20} & =-\kappa \tilde{v}_{10}, \\
\mathrm{i} \omega b_{2} \tilde{u}_{10}-\kappa \tilde{u}_{20} & =-\kappa \tilde{v}_{20} .
\end{aligned}
$$

In the same way as in Section 3.3 we can determine $-\kappa \tilde{v}_{10},-\kappa \tilde{v}_{20}$ by solving the equations (4.14) for $\tilde{v}_{1}, \tilde{v}_{2}$ and reduce the problem (4.11)-(4.12) to the previous problem (4.1)-(4.3). Thus we obtain the same estimates. 


\subsection{General systems. The Cauchy problem}

We consider the Cauchy problem for the homogeneous system of the introduction

$$
u_{t t}=P_{0}(D) u, \quad t \geq 0, x \in \mathbb{R}^{r}:-\infty<x_{j}<\infty, j=1,2, \ldots, r
$$

were

$$
P_{0}(D)=A_{1} D_{1}^{2}+\sum_{j=2}^{r} B_{j} D_{j}^{2} .
$$

$A_{1}=A_{1}^{*}>0, B_{j}=B_{j}^{*}>0$, are positive definite symmetric $n \times n$ matrices and $u$ is a vector valued function with $n$ components.

At $t=0$ we give initial data

$$
u(x, 0)=f_{1}(x), \quad u_{t}(x, 0)=f_{2}(x) .
$$

Also, $f_{1}, f_{2}$ are smooth functions with compact support.

We want to show that the problem is well posed. Fourier transform with respect to $x$ gives us

$$
\hat{u}_{t t}=-\hat{P}_{0}(\omega) \hat{u}, \quad \hat{P}_{0}(\omega)=A_{1} \omega_{1}^{2}+\sum_{j=2}^{r} B_{j} \omega_{j}^{2}, \quad \hat{P}_{0}(\omega)=\hat{P}_{0}^{*}(\omega)>0
$$

and

$$
\hat{u}(\omega, 0)=\hat{f}_{1}(\omega), \quad \hat{u}_{t}(\omega, 0)=\hat{f}_{2}(\omega)
$$

Introducing a new variable

$$
\hat{u}_{t}=\hat{P}_{0}^{1 / 2} \hat{v}
$$

gives us

$$
\left(\begin{array}{l}
\hat{u} \\
\hat{v}
\end{array}\right)_{t}=\left(\begin{array}{cc}
0 & \hat{P}_{0}^{1 / 2} \\
-\hat{P}_{0}^{1 / 2} & 0
\end{array}\right)\left(\begin{array}{l}
\hat{u} \\
\hat{v}
\end{array}\right)
$$

Therefore we obtain

$$
\frac{\partial}{\partial t}\left(|\hat{u}|^{2}+|\hat{v}|^{2}\right)=0
$$

i.e.

$$
\|\hat{u}(\cdot, t)\|^{2}+\|\hat{v}(\cdot, t)\|^{2}=\|\hat{u}(\cdot, 0)\|^{2}+\|\hat{v}(\cdot, 0)\|^{2} .
$$

This energy estimate shows that the Cauchy problem is well posed.

\subsection{The resolvent equation}

Consider the Cauchy problem for the inhomogeneous system (4.16) with $F(x, t) \in \mathrm{C}_{0}^{\infty}$ and with homogeneous initial data $f_{1}=f_{2}=0$.

$$
u_{t t}=P_{0}(D) u+F
$$

Fourier transform with respect to $x$ and Laplace transform with respect to time gives us the resolvent equation

$$
\left(s^{2} I+|\omega|^{2} \hat{P}_{0}\left(\omega^{\prime}\right)\right) \hat{u}=\hat{F}, \quad s=\mathrm{i} \xi+\eta, \eta>0, \omega^{\prime}=\omega /|\omega| .
$$

Since $P_{0}=P_{0}^{*}>0$, there is a unitary transformation which transforms (4.20) to diagonal form

$$
\left(s^{2}+|\omega|^{2} \mu_{j}\right) \tilde{u}_{j}=\tilde{F}_{j}, j=1,2, \ldots, n, \quad \hat{u}=S_{1} \tilde{u}, \hat{F}=S_{1} \tilde{F},
$$

i.e.,

$$
\left(s+\mathrm{i}|\omega| \sqrt{\mu_{j}}\right)\left(s-\mathrm{i}|\omega| \sqrt{\mu_{j}}\right) \tilde{u}_{j}=\tilde{F}_{j} .
$$


Without restriction we can assume that $\xi>0$. Then

$$
\begin{aligned}
& |s+\mathrm{i}| \omega\left|\sqrt{\mu_{j}}\right|=\sqrt{|\xi+| \omega\left|\sqrt{\mu_{j}}\right|^{2}+\eta^{2}} \geq \sqrt{|s|^{2}+|\omega|^{2} \mu_{j}}, \\
& |s-\mathrm{i}| \omega\left|\sqrt{\mu_{j}}\right|=\sqrt{|\xi-| \omega\left|\sqrt{\mu_{j}}\right|^{2}+\eta^{2}} \geq \eta=\operatorname{Re} s .
\end{aligned}
$$

Therefore

$$
\left|\tilde{u}_{j}\right| \leq \frac{\left|\tilde{F}_{j}\right|}{\sqrt{|s|^{2}+|\omega|^{2} \mu_{j}} \operatorname{Re} s} .
$$

Choosing $\operatorname{Im} s=\mathrm{i}|\omega| \sqrt{\mu_{j}}$ shows that the estimate is sharp.

We have proved:

Theorem 4.1. There is a constant $K$ which does not depend on $\omega$ such that the resolvent estimate

$$
|\hat{u}(\omega, s)| \leq \frac{K|\hat{F}|}{\sqrt{|s|^{2}+|\omega|^{2}} \operatorname{Re} s}
$$

holds.

The last estimate shows that we 'gain' one derivative, i.e., if the forcing $\in \mathrm{H}^{p}$, then the solution $\in \mathrm{H}^{p+1}$. Therefore we can prove that the Cauchy problem is stable against lower order perturbations.

Theorem 4.2. Consider the Cauchy problem with homogeneous initial data for

$$
u_{t t}=\left(P_{0}(D)+P_{1}(D)\right) u+F .
$$

Here $P_{1}(D)$ represents a general first order operator. There is an $\eta_{0}>0$ such that the estimate (4.21) holds for $\eta=\operatorname{Re} s>\eta_{0}$.

Proof. We consider $P_{1}(D) u$ as part of the forcing. Then (4.21) gives us

$$
|\hat{u}(\omega, s)| \leq \frac{K}{\operatorname{Re} s} \frac{\left|P_{1}(\mathrm{i} \omega, s) \hat{u}\right|}{\sqrt{|s|^{2}+|\omega|^{2}}}+\frac{K}{\operatorname{Re} s} \frac{|\hat{F}|}{\sqrt{|s|^{2}+|\omega|^{2}}} .
$$

Since $\frac{\left|P_{1}(\mathrm{i} \omega, s)\right|}{\sqrt{|s|^{2}+|\omega|^{2}}}$ is uniformly bounded, we choose $\eta_{0}$ such that

$$
\frac{K}{\eta_{0}} \frac{\left|P_{1}(\mathrm{i} \omega, s) \hat{u}\right|}{\sqrt{|s|^{2}+|\omega|^{2}}} \leq \frac{1}{2}|\hat{u}(\omega, s)|
$$

Then the desired estimate follows.

We can write the resolvent equation also as a first order system. We Fourier transform (4.19) with respect to $x_{-}=\left(x_{2}, \ldots, x_{r}\right)$ and Laplace transform it with respect to $t$. Then we obtain

$$
\begin{gathered}
A_{1} D_{1}^{2} \hat{u}=\left(s^{2} I+B\left(\omega_{-}\right)\right) \hat{u}-\hat{F}, \quad B\left(\omega_{-}\right)=\sum_{j=2}^{r} B_{j} \omega_{j}^{2} \\
\hat{u}=\hat{u}\left(x_{1}, \omega_{-}, s\right) \in \mathrm{L}^{2}\left(0 \leq x_{1}<\infty\right) .
\end{gathered}
$$

Since $A_{1}>0$, there is a constant $\sigma>0$ such that $A_{1}=A_{1}^{*} \geq \sigma I>0$. Introducing a new variable by

$$
D_{1} \hat{u}=\sqrt{|s|^{2}+\left|\omega_{-}\right|^{2}} \hat{v}
$$


we obtain the first order system

$$
D_{1}\left(\begin{array}{l}
\hat{u} \\
\hat{v}
\end{array}\right)=M\left(s, \omega_{-}\right)\left(\begin{array}{l}
\hat{u} \\
\hat{v}
\end{array}\right)+\frac{1}{\sqrt{|s|^{2}+\left|\omega_{-}\right|^{2}}}\left(\begin{array}{c}
0 \\
-A_{1}^{-1} \hat{F}
\end{array}\right)
$$

where

$$
M=M\left(s, \omega_{-}\right)=\left(\begin{array}{cc}
0 & \sqrt{|s|^{2}+\left|\omega_{-}\right|^{2}} I \\
\frac{A_{1}^{-1}\left(s^{2} I+B\left(\omega_{-}\right)\right)}{\sqrt{|s|^{2}+\left|\omega_{-}\right|^{2}}} & 0
\end{array}\right) .
$$

The eigenvalues $\kappa$ of $M$ are solutions of

$$
A_{1} \kappa^{2} \varphi_{0}=\left(B\left(\omega_{-}\right)+s^{2} I\right) \varphi_{0}
$$

Lemma 4.3. For $\operatorname{Re} s>0$, there are no $\kappa_{j}$ with $\operatorname{Re} \kappa_{j}=0$. Also, there are exactly $n$ eigenvalues, counted according to their multiplicity with $\operatorname{Re} \kappa<0$ and, therefore, $n$ eigenvalues with $\operatorname{Re} \kappa>0$.

Proof. Assume there exists a $\kappa=\mathrm{i} \omega_{1}$ which is purely imaginary. Then, by (4.20),

$$
\left(s^{2} I-\hat{P}_{0}\left(\mathrm{i} \omega_{1}, \mathrm{i} \omega_{-}\right)\right) \varphi_{0}=0
$$

has a nontrivial solution, i.e., $s^{2}$ is an eigenvalue of $\hat{P}_{0}(\mathrm{i} \omega) . \hat{P}_{0}(\mathrm{i} \omega)<0$ implies that $s^{2}$ is real and negative which is a contradiction with $\operatorname{Re} s>0$.

The solutions of (4.24) are continuous functions of $\omega_{-}$. Therefore the number of $\kappa$ with $\operatorname{Re} \kappa<0$ does not depend on $\omega_{-}$and we can assume that $\omega_{-}=0$. Then (4.24) reduces to

$$
\left(s^{2} I-A_{1} \kappa^{2}\right) \varphi_{0}=0 .
$$

Since, by assumption, $A_{1}$ has positive eigenvalues $\mu_{j}$ and a complete system of eigenvectors, we can transform (4.26) into $n$ scalar equations

$$
\left(s^{2}-\mu_{j} \kappa^{2}\right) u_{j}=0, \quad j=1,2, \ldots, n,
$$

i.e.,

$$
\kappa= \pm s / \sqrt{\mu_{j}}, \quad \mu_{j}>0, j=1,2, \ldots n, \operatorname{Re} s>0
$$

This proves the lemma.

By Schur's lemma, there exists a unitary transformation $U=U\left(s, \omega_{-}\right)$such that

$$
U^{*}\left(s, \omega_{-}\right) M\left(s, \omega_{-}\right) U\left(s, \omega_{-}\right)=\left(\begin{array}{cc}
M_{11} & M_{12} \\
0 & M_{22}
\end{array}\right),
$$

where the eigenvalues $\kappa_{j 1}, \kappa_{j 2}$ of $M_{11}$ and $M_{22}$ satisfy $\operatorname{Re} \kappa_{j 1}<0$, Re $\kappa_{j 2}>0$, respectively, for Re $s>0$. Clearly, the transformed equation (4.23) can be solved uniquely for $\operatorname{Re} s>0$.

Using (4.21), we shall now derive estimates for the solutions of (4.23). To accomplish this we consider a more general forcing. We replace

$$
\frac{1}{\sqrt{|s|^{2}+\left|\omega_{-}\right|^{2}}}\left(\begin{array}{c}
0 \\
A_{1}^{-1} \hat{F}
\end{array}\right) \quad \text { by } \quad\left(\begin{array}{c}
\hat{F}_{1} \\
\hat{F}_{2}
\end{array}\right), \quad \hat{F}_{j}=\hat{F}_{j}\left(x_{1}, \omega_{-}, s\right) \text {. }
$$


We Fourier transform (4.23) with respect to $x_{1}$ and consider

$$
\begin{aligned}
& \mathrm{i} \omega_{1} \hat{u}=\sqrt{|s|^{2}+\left|\omega_{-}\right|^{2}} \hat{v}-\hat{F}_{1}, \\
& \mathrm{i} \omega_{1} \hat{v}=\frac{A_{1}^{-1}\left(s^{2} I+B\left(\omega_{-}\right)\right)}{\sqrt{|s|^{2}+\left|\omega_{-}\right|^{2}}} \hat{u}-\hat{F}_{2} .
\end{aligned}
$$

Eliminating $\hat{v}$ gives us

$$
\left(s^{2} I+|\omega|^{2} \hat{P}_{0}\left(\omega^{\prime}\right)\right) \hat{u}=\mathrm{i} \omega_{1} A_{1} \hat{F}_{1}+\sqrt{|s|^{2}+\left|\omega_{-}\right|^{2}} A_{1} \hat{F}_{2} .
$$

Therefore, by (4.21), we obtain the estimate

$$
\begin{aligned}
|\hat{u}(\omega, s)| & \leq K \frac{\left|\mathrm{i} \omega_{1} \hat{F}_{1}+\sqrt{|s|^{2}+\left|\omega_{-}\right|^{2}} \hat{F}_{2}\right|}{\sqrt{|s|^{2}+|\omega|^{2}} \operatorname{Re} s} \\
& \leq K \frac{\left|\hat{F}_{1}\right|+\left|\hat{F}_{2}\right|}{\operatorname{Re} s}, \quad \hat{F}_{j}=\hat{F}_{j}\left(\omega_{1}, \omega_{-}, s\right) .
\end{aligned}
$$

Eliminating $\hat{u}$, we obtain the same estimate for $\hat{v}$. Therefore we have proved

Lemma 4.4. There exists a constant $K>0$ such that, for all $\omega_{1}, \omega_{-}, s$,

$$
\left|\left(M\left(s, \omega_{-}\right)-\mathrm{i} \omega_{1} I\right)^{-1}\right| \leq \frac{2 K}{\operatorname{Re} s} .
$$

In particular, the eigenvalues $\kappa$ of $M\left(s, \omega_{-}\right)$satisfy

$$
|\operatorname{Re} \kappa| \geq \frac{\operatorname{Re} s}{2 K} .
$$

Using scaled variables

$$
s^{\prime}=s / \sqrt{|s|^{2}+\left|\omega_{-}\right|^{2}}, \quad \omega^{\prime}=\omega / \sqrt{|s|^{2}+\left|\omega_{-}\right|^{2}}, \quad M^{\prime}\left(s^{\prime}, \omega^{\prime}\right)=\left(\begin{array}{cc}
0 & I \\
A_{1}^{-1}\left(s^{\prime 2} I+\beta\left(\omega_{-}^{\prime}\right)\right) & 0
\end{array}\right),
$$

we can write $(4.28),(4.29)$ in the form

$$
\begin{aligned}
\left|\left(M^{\prime}\left(s^{\prime}, \omega_{-}^{\prime}\right)-\mathrm{i} \omega_{1}^{\prime} I\right)^{-1}\right| & \leq \frac{2 K}{\operatorname{Re} s^{\prime}}, \\
\left|\operatorname{Re} \kappa^{\prime}\right| & \geq \frac{\operatorname{Re} s^{\prime}}{2 K} .
\end{aligned}
$$

\subsection{Reduction to a first order system of pseudo-differential equations}

Now we consider the general halfplane problem (2.1)-(2.3) with homogeneous initial data $f_{1}(x)=f_{2}(x)=$ 0 , coupled to the boundary conditions (2.3). We Fourier transform the problem with respect to $x_{-}=$ $\left(x_{2}, x_{3}, \ldots, x_{r}\right)$ and Laplace transform it with respect to $t$ and obtain (4.22) coupled to the boundary condition

$$
\hat{u}_{x}+\left(C_{0} s+\sum_{j=2}^{r} C_{j} \omega_{j}\right) \hat{u}=\hat{g}, \quad \hat{u}=\hat{u}\left(0, \omega_{-}, s\right), \quad \hat{g}=\hat{g}\left(\omega_{-}, s\right) .
$$

As in Section 3 we have to assume that there are no simple wave solutions for $\operatorname{Re} s>0$ i.e., that the eigenvalue problem consisting of the homogeneous equations (4.22) and (4.33) have no eigenvalues $s$ with Re $s>0$, otherwise the problem is not well posed. 
Now we introduce new variables by

$$
\hat{u}=A_{1}^{-1 / 2} \tilde{u}_{1}, \quad D_{1} \tilde{u}=\sqrt{|s|^{2}+\left|\omega_{-}\right|^{2}} \tilde{v}
$$

and obtain a normalised version of (4.23)

$$
D_{1}\left(\begin{array}{c}
\tilde{u} \\
\tilde{v}
\end{array}\right)=\sqrt{|s|^{2}+\left|\omega_{-}\right|^{2}} \tilde{M}\left(\begin{array}{l}
\tilde{u} \\
\tilde{v}
\end{array}\right)+\frac{1}{\sqrt{|s|^{2}+\left|\omega_{-}\right|^{2}}}\left(\begin{array}{c}
0 \\
-A_{1}^{-1 / 2} \hat{F}
\end{array}\right)
$$

where

$$
H\left(s^{\prime}, \omega^{\prime}\right)=A_{1}^{-1 / 2}\left(s^{\prime 2} I+B\left(\omega_{-}^{\prime}\right)\right) A^{-1 / 2}, \quad \tilde{M}=\left(\begin{array}{cc}
0 & I \\
H & 0
\end{array}\right),
$$

and $s^{\prime}=s / \sqrt{|s|^{2}+\left|\omega_{-}\right|^{2}}, \omega_{-}^{\prime}=\omega_{-} / \sqrt{|s|^{2}+\left|\omega_{-}\right|^{2}}$ are scaled variables.

Instead of (4.24) we obtain now

$$
\begin{aligned}
\psi & =\kappa^{\prime} \varphi, \\
H\left(s^{\prime}, \omega^{\prime}\right) \varphi & =\kappa^{\prime} \psi,
\end{aligned}
$$

i.e.,

$$
\kappa^{\prime 2} \varphi=A_{1}^{-1 / 2}\left(s^{\prime 2} I+B\left(\omega_{-}^{\prime}\right)\right) A_{1}^{-1 / 2} \varphi, \quad \kappa^{\prime}=\frac{\kappa}{\sqrt{|s|^{2}+\left|\omega_{-}\right|^{2}}}
$$

(4.37) is a normalized form of (4.24).

Lemmas 4.3 and 4.4 are crucial because they garantee that we can use the classical theory (see Lemmas 2.1 and 2.2 in [3]). In particular, as in Section 3, away from any eigenvalue or generalised eigenvalue $s$, with $\operatorname{Re} s>0$, the strong estimate of definition 2.5 holds. Thus we need only to consider the estimates in the neigbourhood of generalised eigenvalues.

Also, for Re $s>0$ we can use Schur's lemma to transform $\tilde{M}$ to the upper triangular form (4.27) separating the eigenvalues with $\operatorname{Re} \kappa_{j}<0$ and $\operatorname{Re} \kappa_{j}>0$ respectively. Then we use the technique of Section 3 to estimate the solutions. This becomes particularly simple if there is a standard energy estimate and we need only to show that the problem is boundary stable.

Finally, we want to show that in the neighbourhood of generalised eigenvalues our problem behaves like wave equations.

We shall now derive a normal form of $\tilde{M}$ for $s_{0}^{\prime}=\mathrm{i} \xi_{0}^{\prime}$. We have

Lemma 4.5. Let $s_{0}^{\prime}=\mathrm{i} \xi_{0}^{\prime}, \omega_{-0}^{\prime}$ be a generalised eigenvalue. $H\left(\omega_{0}^{\prime}, \mathrm{i} \xi_{0}^{\prime}\right)$ is symmetric and there is a unitary transformation $U$ such that

$$
U^{*} H U=\left(\begin{array}{cc}
H_{11} & 0 \\
0 & H_{22}
\end{array}\right)
$$

where

$$
\begin{aligned}
& H_{11}=\left(\begin{array}{cccc}
\kappa_{1}^{\prime 2} & & & 0 \\
& \kappa_{2}^{\prime 2} & & \\
& & \ddots & \\
& & & \kappa_{m}^{\prime 2}
\end{array}\right), \quad \kappa_{1}^{\prime 2} \geq \kappa_{2}^{\prime 2} \geq \ldots \geq \kappa_{m}^{\prime 2}>0, \\
& H_{22}=\left(\begin{array}{cccc}
\kappa_{m+1}^{\prime 2} & & & 0 \\
& \kappa_{m+2}^{\prime 2} & & \\
& & \ddots & \\
& & & \kappa_{n}^{\prime 2}
\end{array}\right), \quad 0 \geq \kappa_{m+1}^{\prime 2} \geq \kappa_{m+2}^{\prime 2} \geq \ldots \geq \kappa_{n}^{\prime 2}
\end{aligned}
$$


where $\kappa_{j}^{\prime}= \pm \kappa_{j}^{\prime}\left(\omega_{-0}^{\prime}, \mathrm{i} \xi_{0}^{\prime}\right)$ are the eigenvalues of $(4.37) . \kappa_{m+1}^{\prime 2}=0$ if and only if

$$
\xi_{0}^{\prime 2}=\beta_{j}\left|\omega_{-0}^{\prime}\right|^{2} \quad \text { is an eigenvalue of } B\left(\omega_{-0}^{\prime}\right), \quad 0<\beta_{\min } \leq \beta_{j} \leq \beta_{\max }, j=1,2, \ldots, n \text {. }
$$

Also,

$$
\text { if } \xi_{0}^{\prime 2}<\beta_{\min }\left|\omega_{-0}^{\prime}\right|^{2}, \quad \text { then all } \kappa_{j}^{\prime 2}>0
$$

Proof. Since $H\left(\omega_{-0}^{\prime}, \mathrm{i} \xi_{0}^{\prime}\right)$ is symmetric, we obtain (4.38)-(4.40).

If $\xi_{0}^{\prime 2}>\beta_{\max }\left|\omega_{-0}^{\prime}\right|^{2}$, then $H$ is negative definite and all $\kappa_{j}^{\prime 2}<0$. Correspondingly, if $\xi_{0}^{\prime 2}<\beta_{\min }\left|\omega_{-0}^{\prime}\right|^{2}$, then all $\kappa_{j}^{\prime 2}>0$. If $\kappa^{\prime 2}=0$, then (4.37) becomes

$$
\left(B\left(\omega_{-0}^{\prime}\right)-\xi_{0}^{\prime 2} I\right) \varphi=0,
$$

i.e., $\xi_{0}^{\prime 2}$ must be an eigenvalue of $B\left(\omega_{-0}^{\prime}\right)$. Clearly, the reverse is also true. If $\xi_{0}^{\prime 2}$ is an eigenvalue of $B\left(\omega_{-0}^{\prime}\right)$, then there is a $\kappa^{\prime 2}=0$. This proves the lemma.

To simplify the arguments we shall make a strong assumption which we shall relax at the end of the section.

Assumption 4.6. The eigenvalues $\kappa_{j}^{\prime 2}$ are distinct.

In this case we can choose $U=U\left(\omega_{-}^{\prime}, \xi^{\prime}\right)$ as a smooth function of $\omega_{-}^{\prime}, \xi^{\prime}$ in a neighborhood of $\omega_{-0}^{\prime}, \xi_{0}^{\prime}$. Also there is a constant $d_{0}>0$ such that, in the whole neighborhood,

$$
\left|\kappa_{j}^{\prime 2}-\kappa_{i}^{\prime 2}\right| \geq d_{0} \quad \text { for all } i, j \text { with } \quad i \neq j .
$$

Finally, we make the perturbation $s^{\prime}=\mathrm{i} \xi^{\prime}+\eta^{\prime},-\eta_{0}^{\prime} \leq \eta^{\prime} \leq \eta_{0}^{\prime}, \eta_{0}^{\prime} \ll 1$. We have

$$
U^{*}\left(\omega_{-}^{\prime}, \xi^{\prime}\right) H\left(\omega_{-}^{\prime}, s^{\prime}\right) U\left(\omega_{-}^{\prime}, \xi^{\prime}\right)=U^{*}\left(\omega_{-}^{\prime}, \xi^{\prime}\right) H\left(\omega_{-}^{\prime}, \mathrm{i} \xi^{\prime}\right) U\left(\omega_{-}^{\prime}, \xi^{\prime}\right)+U^{*}\left(\omega_{-}^{\prime}, \xi^{\prime}\right) A^{-1} U\left(\omega_{-}^{\prime}, \xi^{\prime}\right)\left(2 \mathrm{i} \xi^{\prime} \eta^{\prime}+\eta^{2}\right) .
$$

Since $U^{*} A^{-1} U$ is strictly positive definite, its diagonal elements $a_{j j}>0$ are positive. By using well known algebraic results (Gershgorin's theorem) this gives us

Lemma 4.7. For sufficiently small $\eta_{0}^{\prime}$ which depends only on $A^{-1}$ and (4.42), there exists a smooth nonsingular transformation $S=I+\eta^{\prime} S_{1}\left(\omega_{-}^{\prime}, s^{\prime}\right)$ such that

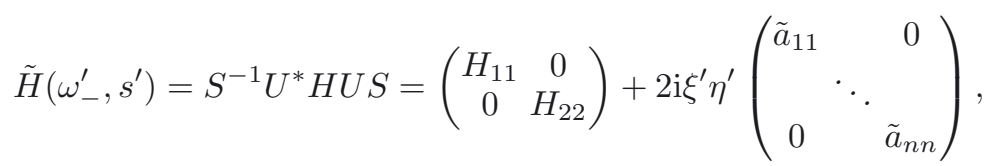

where $\tilde{a}_{j j}=a_{j j}+\mathcal{O}\left(\eta^{\prime}\right)>0$ and $H_{11}, H_{22}$ are given as before but with distinct eigenvalues.

Now we can construct the normal form for the resolvent equation (4.35). We introduce new variables by

$$
\tilde{u}=A_{1}^{-1 / 2} U S \tilde{\tilde{u}}, \quad \tilde{v}=A_{1}^{-1 / 2} U S \tilde{\tilde{v}}
$$

and after a permutation we obtain

Theorem 4.8. In a neighborhood of $\omega_{-0}^{\prime}, \xi_{0}^{\prime}$ the resolvent equation (4.35) can be transformed smoothly into

$$
D_{1}\left(\begin{array}{c}
\tilde{\tilde{u}} \\
\tilde{\tilde{v}}
\end{array}\right)=\sqrt{|s|^{2}+\left|\omega_{-}\right|^{2}}\left(\begin{array}{cc}
0 & I \\
\tilde{H}\left(\omega_{-}^{\prime}, s^{\prime}\right) & 0
\end{array}\right)\left(\begin{array}{c}
\tilde{\tilde{u}} \\
\tilde{\tilde{v}}
\end{array}\right)+\frac{1}{\sqrt{|s|^{2}+\left|\omega_{-}\right|^{2}}}\left(\begin{array}{l}
0 \\
\tilde{\tilde{F}}
\end{array}\right)
$$

where $\tilde{\tilde{F}}=-S^{-1} U^{*} A_{1}^{1 / 2} \hat{F}$. By (4.44), the system (4.45) is composed of $2 \times 2$ systems

$$
\left(\begin{array}{c}
\tilde{\tilde{u}}_{j} \\
\tilde{v}_{j}
\end{array}\right)_{x}=\sqrt{|s|^{2}+\left|\omega_{-}\right|^{2}}\left(\begin{array}{cc}
0 & 1 \\
\tilde{\kappa}_{j}^{\prime 2}\left(\omega^{\prime}, s^{\prime}\right) & 0
\end{array}\right)\left(\begin{array}{c}
\tilde{\tilde{u}}_{j} \\
\tilde{\tilde{v}}_{j}
\end{array}\right)+\frac{1}{\sqrt{|s|^{2}+\left|\omega_{-}\right|^{2}}}\left(\begin{array}{c}
0 \\
\tilde{\tilde{F}}_{j}
\end{array}\right)
$$

where

$$
\tilde{\kappa}_{j}^{\prime 2}(\omega, s)=\kappa_{j}^{\prime 2}\left(\omega_{-}^{\prime}, \mathrm{i} \xi^{\prime}\right)+2 i \tilde{a}_{j j} \xi^{\prime} \eta^{\prime} .
$$

Clearly, the $2 \times 2$ blocks have the form (3.13) of Section 3 . 
There are no difficulties to generalize the results to the case that the $\kappa_{j}^{2}$ have constant multiplicity (this was done in [1] for first orders systems).

\section{NUMERICAL EXPERIMENTS}

In this section we numerically solve the strip problem for the scalar wave equation

$$
u_{t t}=u_{x x}+u_{y y}+F(x, y, t), \quad 0 \leq x \leq 1,0 \leq y \leq 1, t \geq 0,
$$

with 1-periodic solutions in the $y$-direction,

$$
u(x, y, t)=u(x, y+1, t), \quad 0 \leq x \leq 1, t \geq 0,
$$

subject to initial conditions,

$$
u(x, y, 0)=f_{1}(x, y), \quad u_{t}(x, y, 0)=f_{2}(x, y), \quad 0 \leq x \leq 1,0 \leq y \leq 1,
$$

and boundary conditions

$$
\begin{aligned}
u_{x}-b u_{y}=g_{0}(y, t), & x=0,0 \leq y \leq 1, t \geq 0, \\
u_{x}=g_{1}(y, t), & x=1,0 \leq y \leq 1, t \geq 0 .
\end{aligned}
$$

Here $b$ is a constant. We are interested in the three cases $b=0, b$ real, and $b$ purely imaginary, i.e.,

$$
b=\mathrm{i} \beta, \quad \beta \text { real. }
$$

To solve the latter problem using real arithmetic, we introduce real-valued functions $u^{(1)}$ and $u^{(2)}$ such that

$$
u=u^{(1)}+\mathrm{i} u^{(2)} .
$$

Inserting (5.6) into (5.1) leads to the system of scalar wave equations,

$$
\begin{array}{lll}
u_{t t}^{(1)}=u_{x x}^{(1)}+u_{y y}^{(1)}+\operatorname{Re} F(x, y, t), & 0 \leq x \leq 1, \quad 0 \leq y \leq 1, \quad t \geq 0, \\
u_{t t}^{(2)}=u_{x x}^{(2)}+u_{y y}^{(2)}+\operatorname{Im} F(x, y, t), & 0 \leq x \leq 1, \quad 0 \leq y \leq 1, \quad t \geq 0 .
\end{array}
$$

Boundary condition (5.4) can be written as

$$
\begin{aligned}
& u_{x}^{(1)}+\beta u_{y}^{(2)}=\operatorname{Re} g_{0}(y, t), \quad x=0,0 \leq y \leq 1, t \geq 0, \\
& u_{x}^{(2)}-\beta u_{y}^{(1)}=\operatorname{Im} g_{0}(y, t), \quad x=0,0 \leq y \leq 1, t \geq 0,
\end{aligned}
$$

which is of the form (4.3) with $b_{1}=\beta$ and $b_{2}=-\beta$.

We introduce a grid with grid size $h=1 /(N-1)$,

$$
x_{j}=(j-1) h, \quad j=0,1, \ldots, N+1, \quad y_{k}=k h, \quad k=0,1, \ldots, N .
$$

Time is discretized on a uniform grid with time step $\delta_{t}>0, t_{n}=n \delta_{t}, n=0,1,2, \ldots$ and we denote a grid function by

$$
v_{j, k}^{n}=v\left(x_{j}, y_{k}, t_{n}\right) \text {. }
$$

The standard divided difference operators are defined by

$$
D_{+x} v_{j, k}^{n}=\frac{v_{j+1, k}^{n}-v_{j, k}^{n}}{h}, \quad D_{-x} v_{j, k}^{n}=D_{+x} v_{j-1, k}^{n}, \quad D_{0 x}=\frac{1}{2}\left(D_{+x}+D_{-x}\right),
$$

with corresponding notations in the $y$ - and $t$-directions. 
TABLE 1. Max error in the solution as function of the grid size with a traveling wave exact solution.

\begin{tabular}{c|c|c|c}
\hline Case & $h$ & $\left\|u_{w}-v\right\|_{\infty}(t=1)$ & $\left\|u_{w}-v\right\|_{\infty}(t=10)$ \\
\hline$b=0$ & $1 \times 10^{-2}$ & $7.09 \times 10^{-4}$ & $4.69 \times 10^{-4}$ \\
& $5 \times 10^{-3}$ & $1.76 \times 10^{-4}$ & $1.18 \times 10^{-4}$ \\
& $2.5 \times 10^{-3}$ & $4.42 \times 10^{-5}$ & $2.97 \times 10^{-5}$ \\
\hline$b=0.5$ & $1 \times 10^{-2}$ & $7.09 \times 10^{-4}$ & $2.95 \times 10^{-2}$ \\
& $5 \times 10^{-3}$ & $1.76 \times 10^{-4}$ & $7.37 \times 10^{-3}$ \\
& $2.5 \times 10^{-3}$ & $4.42 \times 10^{-5}$ & $1.84 \times 10^{-3}$ \\
\hline$b=i 0.5$ & $1 \times 10^{-2}$ & $1.44 \times 10^{-3}$ & $1.61 \times 10^{-3}$ \\
& $5 \times 10^{-3}$ & $3.61 \times 10^{-4}$ & $4.02 \times 10^{-4}$ \\
& $2.5 \times 10^{-3}$ & $9.03 \times 10^{-5}$ & $1.00 \times 10^{-4}$ \\
\hline
\end{tabular}

Consider the difference approximation

$$
D_{+t} D_{-t} v_{j, k}^{n}=\left(D_{+x} D_{-x}+D_{+y} D_{-y}\right) v_{j, k}^{n}+F\left(x_{j}, y_{k}, t_{n}\right),
$$

subject to boundary conditions

$$
\begin{aligned}
v_{j, 0}^{n}-v_{j, N-1}^{n} & =0, & & j=0,1, \ldots, N+1, \\
v_{j, N}^{n}-v_{j, 1}^{n} & =0, & & j=0,1, \ldots, N+1, \\
D_{0 x} v_{1, k}^{n}-b D_{0 y} v_{1, k}^{n} & =g_{0}\left(y_{k}, t_{n}\right), & & k=1,2, \ldots, N-1, \\
D_{0 x} v_{N, k}^{n} & =g_{1}\left(y_{k}, t_{n}\right), & & k=1,2, \ldots, N-1,
\end{aligned}
$$

for $n=-1,0,1, \ldots$, and initial conditions,

$$
v_{j, k}^{0}=f_{1}\left(x_{j}, y_{k}\right), \quad v_{j, k}^{-1}=f_{2}^{\prime}\left(x_{j}, y_{k}\right), \quad j=0,1, \ldots, N+1, k=0,1, \ldots, N .
$$

When $b=0$, the difference approximation (5.11)-(5.16) satisfies a discrete energy estimate, under the Courant time step restriction

$$
\delta_{t} \leq C h,
$$

and is therefore stable. The energy method can not be used to show stability of the difference approximation when $b$ is non-zero and real, or purely imaginary. However, as we shall see below, our practical experience indicates that the approximation is stable also when $b$ is purely imaginary. When $b$ is non-zero and real, the continuous problem is unstable. In this case, the difference approximation is convergent for short times.

To test the accuracy of the numerical solution, we choose the forcing functions $F, g_{k}$, and $f_{k}$ such that the exact solution becomes the traveling wave

$$
u_{w}(x, y, t)=\sin (2 \pi(x-t)) \sin (2 \pi y) .
$$

For the case $b=\mathrm{i} \beta$, we use the exact solution

$$
\begin{aligned}
& u_{w}^{(1)}(x, y, t)=\sin (2 \pi(x-t)) \sin (2 \pi y), \\
& u_{w}^{(2)}(x, y, t)=\cos (2 \pi(x-t)) \cos (2 \pi y) .
\end{aligned}
$$

Table 1 shows the max norm of the error $u_{w}-v$ at different times for grid sizes $h=10^{-2}, 5 \times 10^{-3}, 2.5 \times 10^{-3}$. All calculations used $\delta_{t}=0.5 h$. The error decreases as $\mathcal{O}\left(h^{2}\right)$ for all three values of $b$, both at time $t=1$ and $t=10$. 

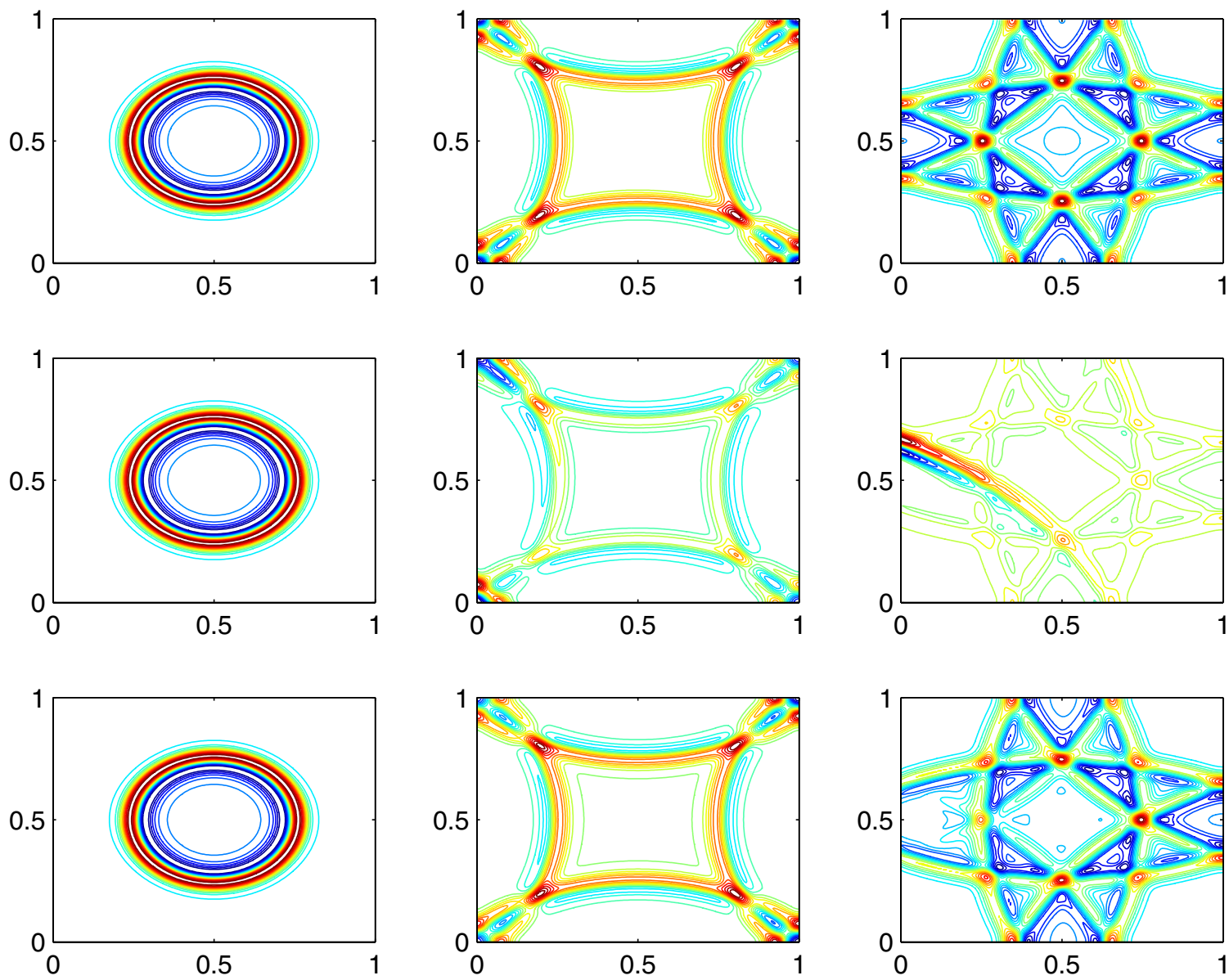

Figure 1. The solution at times $t=0.25$ (left column), $t=0.75$ (middle column), and $t=1.25$ (right column) for $b=0$ (top row), $b=0.5$ (middle row), and $b=i 0.5$ (bottom row). The bottom row is showing the real part of the solution $\left(u^{(1)}\right)$.

To illustrate how the $b$-coefficient in the boundary condition influences the solution, we study the evolution from an initial Gaussian pulse,

$$
f_{1}(x, y)=f_{2}^{\prime}(x, y)=\mathrm{e}^{-(x-0.5)^{2} / L^{2}-(y-0.5)^{2} / L^{2}}, \quad L=0.03,
$$

with homogeneous interior and boundary forcing functions, $F=0, g_{0}=0, g_{1}=0$. In these calculations we use the grid size $h=5 \times 10^{-3}$ and time step $\delta_{t}=2.5 \times 10^{-3}$. The evolution between times $t=0.25$ and $t=1.25$ for the three cases $b=0, b=0.5$, and $b=i 0.5$ is shown in Figure 1 . The solution initially propagates outwards towards the boundary (first column). Before the pulse reaches the boundary, the solutions are identical for all three cases. The middle column in Figure 1 shows the solutions after the initial pulse has reached the boundary, at $t=0.75$. At this time, there are only minor differences between the three solutions. The influence of the boundary condition is becoming more obvious in the right column, corresponding to $t=1.25$. While the differences between $b=0$ and $b=i 0.5$ are still small and located near the left boundary, the case $b=0.5$ has developed a structure near that boundary which is not present for $b=0$, or $b=i 0.5$. For later times, the boundary structure develops into a diagonal streak which extends further and further into the domain until it 

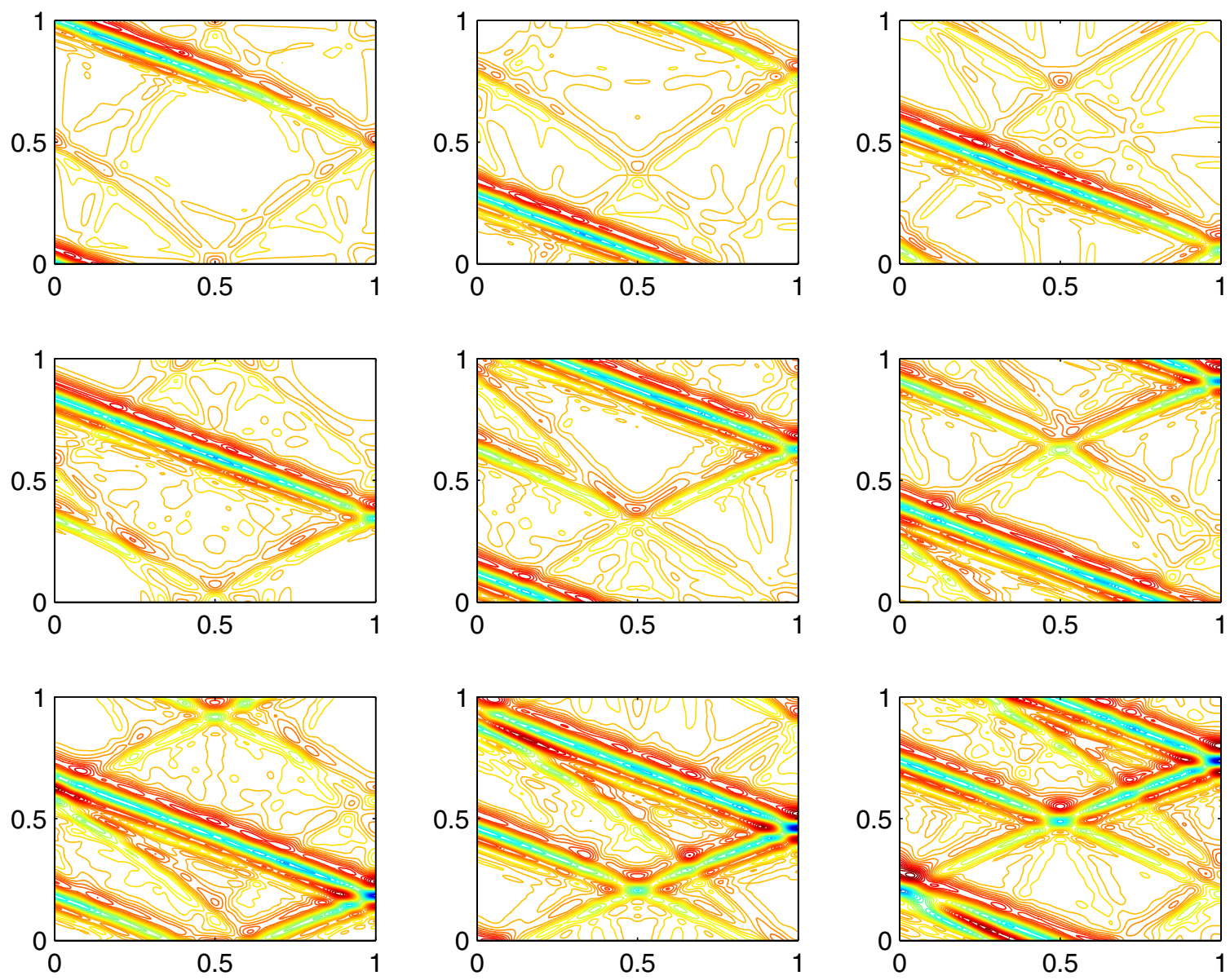

Figure 2. The solution of the unstable case $(b=0.5)$ at times $t=2.5$ to $t=4.5$ in increments of 0.25 , starting in the top left sub-figure and progressing row-wise to the bottom right sub-figure, e.g. $t=2.75$ is in the middle column of the top row.

gets reflected by the Neumann condition on the right boundary, see Figure 2. At later times, the reflected streak develops a new streak, which grows into the domain in the same way. The solution is eventually dominated by these streaks which appear to propagate in the direction $y+0.5 x$. Note that the propagation direction $y+0.5 x$ is consistent with eigenfunction (3.9) since $b=0.5$, see case (4) in Section 3.1.

It is also interesting to monitor the max norm of the solution for longer times when $b=0.5$, see Figure 3 . Note that the solution grows exponentially with time, illustrating the unstable nature of this boundary condition. Also note that the solution is slightly larger on the finer grid. This behavior agrees with the predicted exponential growth proportional to $|\omega|^{t}$, because higher values of $|\omega|$ are captured on the finer grid. Note, however, that this growth is not due to numerical instabilities because the accuracy test shows second order convergence, at least up to $t=10$, see Table 1 .

To more clearly see the difference between the cases $b=0$ and $b=\mathrm{i} \beta$ we take $F=0, g_{0}=g_{1}=0$ and change the initial data to trigger a surface wave,

$$
f_{1}(x, y)=u_{s}(x, y, 0), \quad f_{2}^{\prime}(x, y)=u_{s}\left(x, y,-\delta_{t}\right),
$$




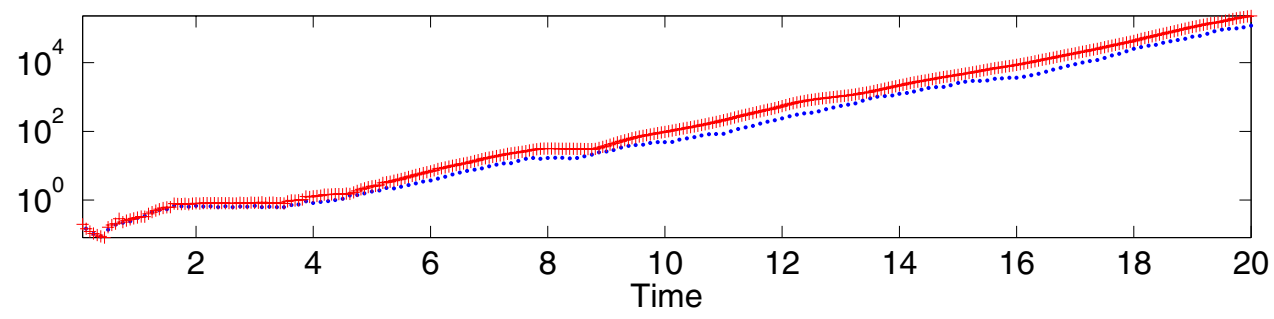

Figure 3. The max norm of the solution for $0 \leq t \leq 20$ for the case $b=0.5$, starting from a Gaussian pulse. The blue dots correspond to grid size $h=10^{-2}$ and the red crosses have $h=5 \times 10^{-3}$.

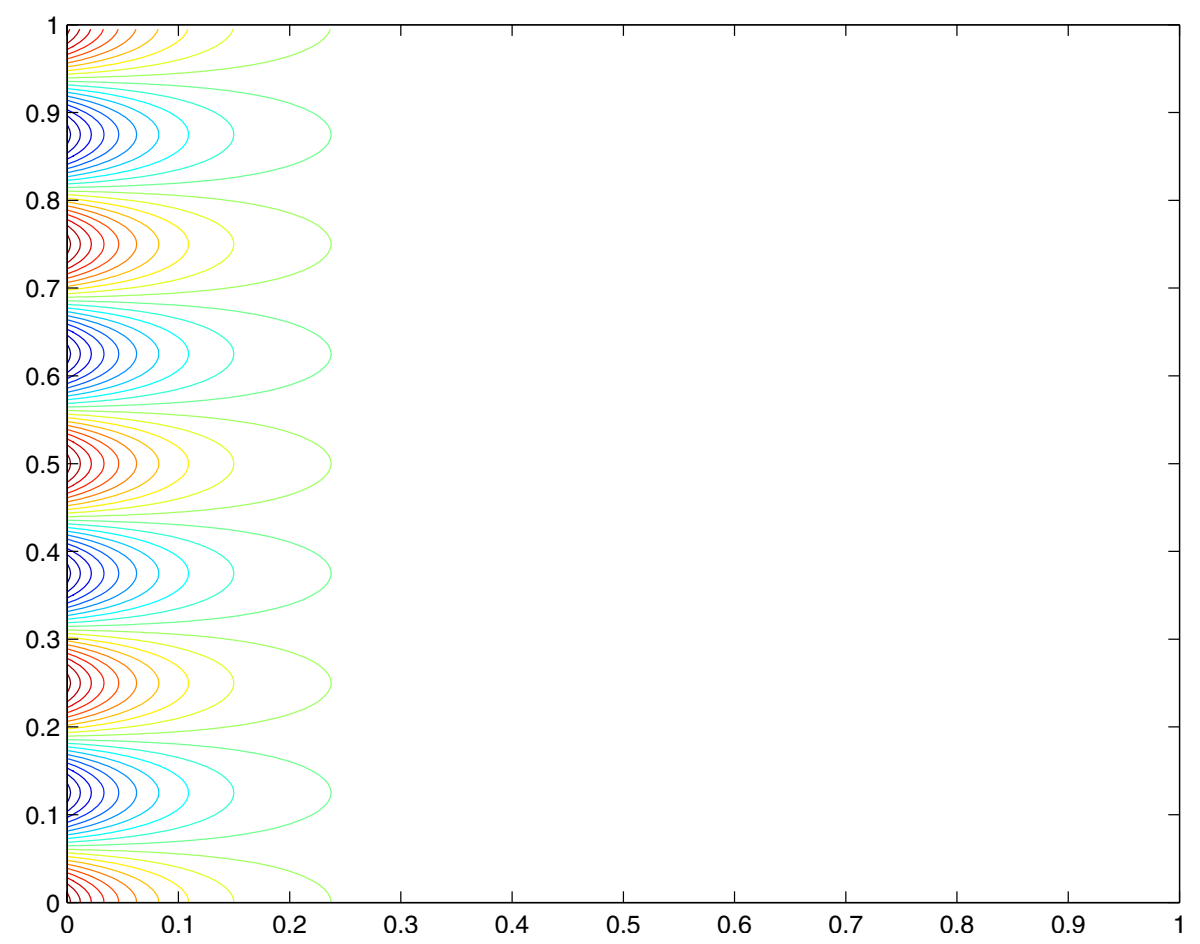

FiguRE 4. The real part of the initial data for the surface wave with $\beta=0.5$ and $\omega_{0}=8 \pi$.

where

$$
u_{s}(x, y, t)=\mathrm{e}^{-\left|\beta \omega_{0}\right| x}\left[\cos \left(\omega_{0}\left(y-\sqrt{1-\beta^{2}} t\right)\right)+\mathrm{i} \sin \left(\omega_{0}\left(y-\sqrt{1-\beta^{2}} t\right)\right)\right], \quad \beta \omega_{0}>0 .
$$

This wave decays exponentially away from the $x=0$ boundary with a harmonic oscillation in $y$, see Figure 4 . The surface wave propagates in the positive $y$-direction with a wave speed proportional to $\sqrt{1-\beta^{2}}$. As $\beta \rightarrow 0$, the surface wave decays slower and slower in the $x$-direction. In the limit $\beta=0$, the amplitude of the wave is constant in $x$ and corresponds to one-dimensional wave propagation in the $y$-direction, consistent with the limiting boundary condition $u_{x}=0$. There are no numerical difficulties in this limit. 
TABLE 2. Max error in the solution as function of the grid size when the exact solution is the surface wave $u_{s}(x, y, t)$.

\begin{tabular}{c|c|c|c}
\hline Case & $h$ & $\left\|u_{s}-v\right\|_{\infty}(t=1)$ & $\left\|u_{s}-v\right\|_{\infty}(t=10)$ \\
\hline$\beta=0.5$ & $1 \times 10^{-2}$ & $2.44 \times 10^{-2}$ & $2.38 \times 10^{-1}$ \\
& $5 \times 10^{-3}$ & $6.35 \times 10^{-3}$ & $6.19 \times 10^{-2}$ \\
& $2.5 \times 10^{-3}$ & $1.60 \times 10^{-3}$ & $1.56 \times 10^{-2}$ \\
\hline$\beta=0.9$ & $1 \times 10^{-2}$ & $6.04 \times 10^{-1}$ & $2.46 \times 10^{-1}$ \\
& $5 \times 10^{-3}$ & $1.58 \times 10^{-1}$ & $1.40 \times 10^{0}$ \\
& $2.5 \times 10^{-3}$ & $4.00 \times 10^{-2}$ & $3.95 \times 10^{-1}$ \\
\hline$\beta=0.99$ & $1 \times 10^{-2}$ & $1.67 \times 10^{0}$ & $1.37 \times 10^{0}$ \\
& $5 \times 10^{-3}$ & $5.20 \times 10^{-1}$ & $1.81 \times 10^{-1}$ \\
& $2.5 \times 10^{-3}$ & $1.44 \times 10^{-1}$ & $1.47 \times 10^{0}$ \\
\hline
\end{tabular}

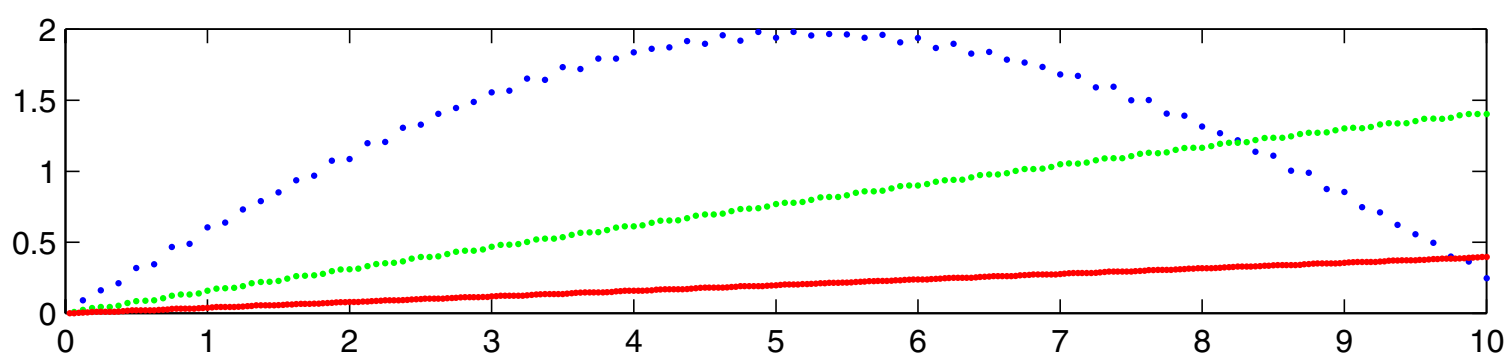

Figure 5. The max norm of the error as function of time for a surface wave with $\beta=0.9$ computed on a grid with $h=10^{-2}$ (blue), $h=5 \times 10^{-3}$ (green), and $h=2.5 \times 10^{-3}$ (red).

The case $|\beta| \rightarrow 1$ is more difficult to solve numerically. Here we study $0.5 \leq \beta<1$ and we use (5.17) as an approximation of the exact solution $\left(u_{s}\right.$ is only exponentially small at $x=1$ and does not exactly satisfy the boundary condition at that boundary). To make sure the amplitude of the surface wave is negligible at the $x=1$ boundary, we choose

$$
\omega_{0}=8 \pi, \quad \mathrm{e}^{-\left|\beta \omega_{0}\right|}=\mathrm{e}^{-4 \pi} \approx 3.48 \times 10^{-6}, \quad \beta=0.5 .
$$

In Table 2 we show the max norm of the error $u_{s}-v$ for different values of $\beta$. The case $\beta=0.5$ shows second order convergence, both at time $t=1$ and $t=10$. As can be expected in wave propagation problems, the error is dominated by the phase error, which explains why it is about 10 times larger at $t=10$ compared to $t=1$. For $\beta=0.9$, the error still converges to second order accuarcy at time $t=1$, but shows an unexpected pattern at time $t=10$. Here the error is larger for the intermediate grid size $h=5 \times 10^{-3}$ than for the coarse grid size $h=10^{-2}$. This behavior is explained by studying the time history of the error, see Figure 5 . For $h=10^{-2}$, the max error occurs at $t \approx 5.5$ when the numerical solution is about 180 degrees out of phase with the exact solution. At later times the error in the numerical solution decreases because it is between 180 and 360 degrees out of phase. The grid with $h=5 \times 10^{-3}$ is barely fine enough to capture the solution at time $t=10$ because the phase error exceeds 90 degrees. As a result we don't see the expected second order convergence when the grid is refined to $h=2.5 \times 10^{-3}$. However, the error at $t=10$ is about 10 times larger than at $t=1$ for the finest grid, which indicates that this resolution is adequate for $\beta=0.9$. The situation is even more dire for $\beta=0.99$. Here the errors at time $t=1$ show a simular behavior as at $t=10$ for $\beta=0.9$, so only the finest grid provides adequate resolution at $t=1$. At $t=10$, the error displays a completely erratic behavior with the largest error for the finest grid. An even finer grid would be necessary to obtain an accurate solution at $t=10$, when $\beta=0.99$. 
So why is it so hard to calculate an accurate numerical solution as $|\beta| \rightarrow 1$ ? The spatial resolution in terms of grid points per wave length only depends on $\omega_{0}$. With $\omega_{0}=8 \pi$, the wave length is $1 / 4$ and grid sizes $h=10^{-2}, 5 \times 10^{-3}, 2.5 \times 10^{-3}$ correspond to 25,50 , and 100 grid points per wave length, respectively. The exponential decay in the $x$-direction only depends weakly on $\beta$ and never exceeds $\mathrm{e}^{-\left|\omega_{0}\right| x}$ for $\beta<1$. Hence the solution varies on the same length scale in the $x$ - and $y$-directions. Furthermore, the temporal resolution in terms of time steps per period only improves as $|\beta| \rightarrow 1$ because the wave speed goes to zero in this limit. We conclude that the numerical difficulties are not due to poor resolution of the solution.

To further analyze the cause of the poor accuracy in the numerical solution for $|\beta| \rightarrow 1$, we decompose the problem (5.1)-(5.5) into two parts,

$$
u(x, y, t)=U(x, y, t)+u^{\prime}(x, y, t),
$$

such that $U$ satisfies a doubly periodic problem on an extended domain,

$$
U_{t t}=U_{x x}+U_{y y}+\tilde{F}(x, y, t), \quad-1 \leq x \leq 2,0 \leq y \leq 1, t \geq 0,
$$

subject to initial conditions,

$$
U(x, y, 0)=\tilde{f}_{1}(x, y), \quad U_{t}(x, y, 0)=\tilde{f}_{2}(x, y), \quad-1 \leq x \leq 2,0 \leq y \leq 1,
$$

and periodic boundary conditions

$$
\begin{aligned}
& U(x, y, t)=U(x, y+1, t), \quad-1 \leq x \leq 2, t \geq 0, \\
& U(x, y, t)=U(x+3, y, t), \quad 0 \leq y \leq 1, t \geq 0 .
\end{aligned}
$$

The interior forcing function and the initial data can be smoothly extended to become 3-periodic in the $x$-direction, without changing them on the original domain,

$$
\tilde{F}(x, y, t)=F(x, y, t), \quad \tilde{f}_{k}(x, y)=f_{k}(x, y), \quad 0 \leq x \leq 1,0 \leq y \leq 1, t \geq 0 .
$$

The problem for $U$ is independent of the $b$-coefficient in the boundary condition and can easily be solved numerically.

The difference $u^{\prime}=u-U$ satisfies the scalar wave equations (5.1)-(5.5) with homogeneous interior forcing, homogeneous initial data, but inhomogeneuos boundary conditions,

$$
\begin{array}{r}
u_{x}^{\prime}-b u_{y}^{\prime}=g_{0}^{\prime}(y, t) \quad x=0,0 \leq y \leq 1, t \geq 0, \\
u_{x}^{\prime}=g_{1}^{\prime}(y, t) \quad x=1,0 \leq y \leq 1, t \geq 0 .
\end{array}
$$

The boundary forcing functions depend on $U$ according to

$$
\begin{array}{ll}
g_{0}^{\prime}(y, t)=g_{0}(y, t)-\left(U_{x}(0, y, t)-b U_{y}(0, y, t)\right), & 0 \leq y \leq 1, t \geq 0, \\
g_{1}^{\prime}(y, t)=g_{1}(y, t)-U_{x}(1, y, t), & 0 \leq y \leq 1, t \geq 0 .
\end{array}
$$

The corresponding half-plane problems were analyzed in Section 3.2. The accuracy problems are unlikely to arise from the Neumann boundary condition at $x=1$ since it is independent of the $b$-coefficient. However, the half-plane problem subject to (5.18) satisfies the estimates of Theorem 3.8. Here, $b=\mathrm{i} \beta$ corresponds to case (2), and estimate (3.25) shows that the Laplace-Fourier transform of $u^{\prime}$ satisfies

$$
\left|\tilde{u}^{\prime}(0, \omega, s)\right|^{2} \leq \frac{C \beta^{2}}{1-\beta^{2}} \frac{\left|\tilde{g}_{0}^{\prime}\right|^{2}}{\eta^{2}}, \quad \operatorname{Re} s=\eta>0,
$$


for $(\omega, s)$ in the vicinity of the generalized eigenvalue $s_{0}= \pm \mathrm{i} \sqrt{1-\beta^{2}} \omega_{0}$. In general, the solution becomes unbounded as $|\beta| \rightarrow 1$. The truncation error terms that perturb the numerical solution are therefore amplified by a factor $1 / \sqrt{1-\beta^{2}}$, which explains the poor accuracy in the numerical solution as $|\beta| \rightarrow 1$.

For boundary data $g_{0}(y, t)$ that have a Laplace-Fourier transform that can be written as

$$
\tilde{g}_{0}(\omega, s)=s \tilde{G}(\omega, s),
$$

estimate (5.20) becomes

$$
\left|\tilde{u}^{\prime}(0, \omega, s)\right|^{2} \leq \frac{C \beta^{2}}{1-\beta^{2}} \frac{|s|^{2}|\tilde{G}|^{2}}{\eta^{2}} \approx \frac{C \beta^{2}}{\left|s_{0}\right|^{2} / \omega_{0}^{2}} \frac{|s|^{2}|\tilde{G}|^{2}}{\eta^{2}}=C \beta^{2} \omega_{0}^{2} \frac{|\tilde{G}|^{2}}{\eta^{2}}, \quad s \rightarrow s_{0} .
$$

Hence the $|\beta| \rightarrow 1$ singularity cancels out and the solution is bounded independently of $\beta$. The factor ' $s$ ' on the Laplace transform side corresponds to a time-derivative on the un-transformed side. Hence, the solution is bounded independently of $\beta$ if the boundary forcing can be written as a time-derivative of a function with bounded Laplace-Fourier transform,

$$
g_{0}(y, t)=G_{t}(y, t), \quad G(y, 0)=0, \quad\left|\int_{y=0}^{1} \int_{t=0}^{\infty} \mathrm{e}^{-2 \pi \mathrm{i} \omega y} \mathrm{e}^{-s t} G(y, t) \mathrm{d} t \mathrm{~d} y\right|<\infty, \quad \operatorname{Re} s \geq 0 .
$$

The latter condition is satisfied if $G(y, t)$ is in $L^{1}$, i.e.,

$$
\int_{y=0}^{1} \int_{t=0}^{\infty}|G(y, t)| \mathrm{d} t \mathrm{~d} y<\infty .
$$

To test this theory numerically, we use a homogeneous interior forcing $(F=0)$ and homogeneous initial conditions $\left(f_{1}=f_{2}=0\right)$, homogeneous forcing on the $x=1$ boundary $\left(g_{1}=0\right)$, and consider three different forcing functions on the $x=0$ boundary: $g_{0}^{(1)}(y, t)=G(y, t), g_{0}^{(2)}(y, t)=G_{t}(y, t)$, and $g_{0}^{(3)}(y, t)=G_{t t}(y, t)$. Here we choose $G(y, t)$ to trigger a surface wave:

$$
G(y, t)=u_{s}(0, y, t) \mathrm{e}^{-\left(t / t_{0}-7\right)^{2}}, \quad t_{0}=0.2,
$$

where $u_{s}$ is defined by (5.17). The Gaussian pulse $\exp \left(-\left(t / t_{0}-7\right)^{2}\right)$ decays exponentially fast away from its center at $t=7 t_{0}$. For example, it equals $1.23 \times 10^{-4}$ at $t=7 t_{0} \pm 3 t_{0}$, and $5.24 \times 10^{-22}$ at $t=7 t_{0} \pm 7 t_{0}$. The function $G(y, t)$ satisfies (5.21), so our theory predicts that boundary forcings $g_{0}^{(2)}$ and $g_{0}^{(3)}$ should give solutions that are bounded independently of $\beta$. However, the time-integral of a Gaussian pulse is the error-function (erf), so the boundary forcing $g_{0}^{(1)}$ does not satisfy $(5.21)$.

In the numerical calculations we take $\omega_{0}=8 \pi$ and study the cases $\beta=0.5, \beta=0.9$, and $\beta=0.99$. The grid size and time step are $h=2.5 \times 10^{-3}$ and $\delta_{t}=0.5 h$. The max norm of the solution as function of time is shown in Figure 6. The case $g_{0}^{(1)}=G$ in the top sub-figure illustrates the general case where the solution grows as $|\beta| \rightarrow 1$. Note that estimate (5.20) predicts the solution to grow like $\beta / \sqrt{1-\beta^{2}}$, which means that the solution should be about 3.5 times larger for $\beta=0.99$ than $\beta=0.9$. In the numerical calculation, the max norm of the solution grows from about 0.75 for $\beta=0.9$ to 3.75 for $\beta=0.99$, which is slightly faster than predicted by theory. The case $g_{0}^{(3)}=G_{t t}$ in the bottom sub-figure shows the opposite situation when the solution decays as $\beta \rightarrow 1$ because the forcing function is a second time-derivative of a function with bounded $L^{1}$ norm, corresponding to an $s^{2}$ factor on the Laplace transform side. The intermediate case $g_{0}^{(2)}=G_{t}$ is shown in the middle sub-figure. Here the solution grows between $\beta=0.9$ and $\beta=0.99$, but not as fast as for $g_{0}^{(1)}$. To more closely study the behavior near $\beta=1$, we take $\beta=0.995,0.999$ and 0.9997 corresponding to $\sqrt{1-\beta^{2}} \approx 0.0998,0.0447$ and 0.0244 , respectively. To properly resolve the solution we here use an extra fine grid with $h=1.25 \times 10^{-3}$ and $\delta_{t}=0.5 h$. The max norm of the solutions, shown in Figure 7 , reveal that the solution indeed stays bounded independently of $\beta$, confirming our theory also for boundary forcing $g_{0}^{(2)}=G_{t}$. 

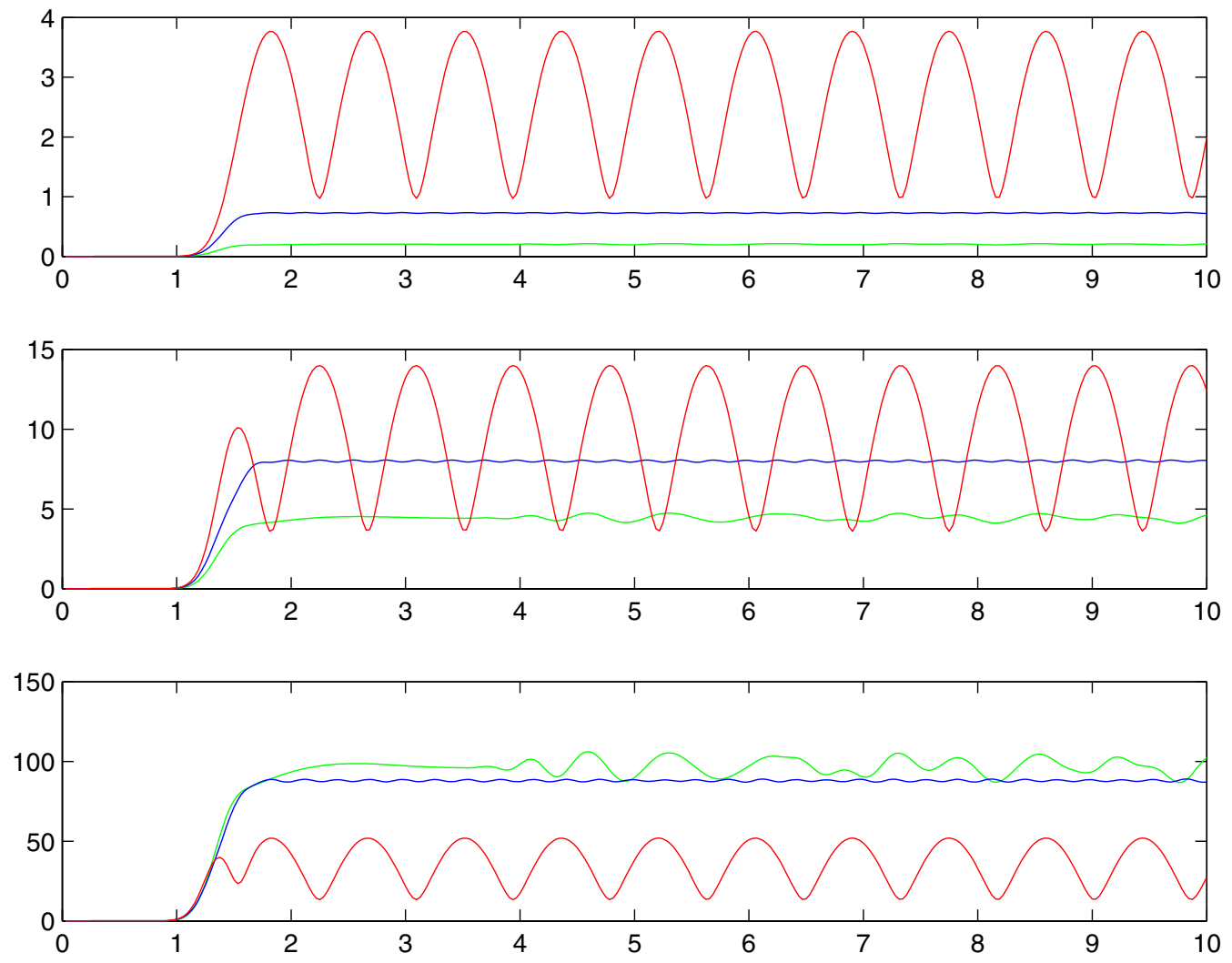

FiguRE 6 . The max norm of the solution as function of time for the boundary forcing functions $g_{0}^{(1)}=G$ (top), $g_{0}^{(2)}=G_{t}$ (middle), and $g_{0}^{(3)}=G_{t t}$ (bottom). In each figure, the green, blue, and red curves correspond to $\beta=0.5, \beta=0.9$, and $\beta=0.99$, respectively.

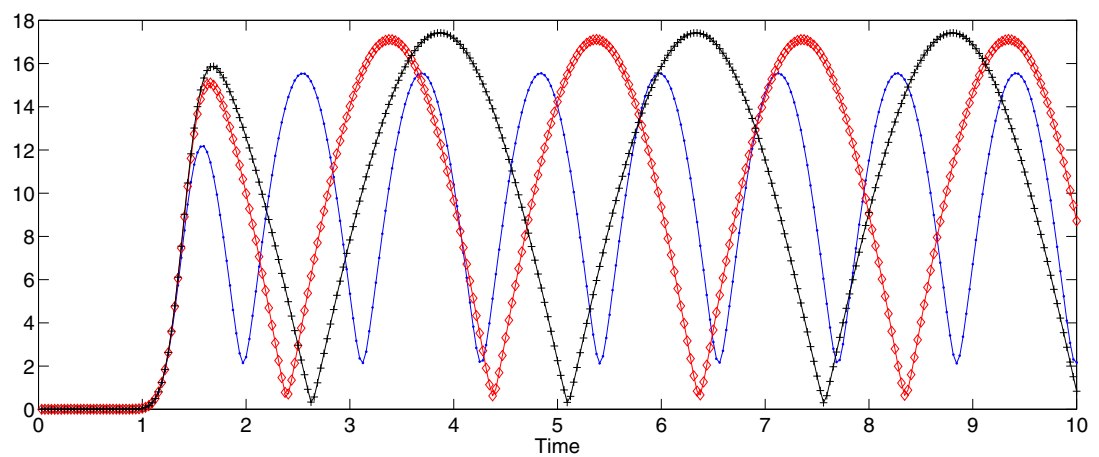

FIgURE 7. The max norm of the solution as function of time for the boundary forcing function $g_{0}^{(2)}=G_{t}$ for $\beta=0.995$ (blue/dots), $\beta=0.999$ (red/diamonds) and $\beta=0.9997$ (black/plusses). 


\section{APPENDIX}

In this appendix we collect a number of auxilary lemmas.

Lemma A.1. The solution of

$$
y_{x}=\lambda y+F, \quad \operatorname{Re} \lambda>0, \quad 0 \leq x<\infty
$$

satisfies the estimate

$$
|y(0)|^{2} \leq \frac{1}{2 \operatorname{Re} \lambda}\|F\|^{2}, \quad\|y\|^{2} \leq \frac{1}{(\operatorname{Re} \lambda)^{2}}\|F\|^{2}, \quad\|F\|^{2}=\int_{0}^{\infty}|F|^{2} \mathrm{~d} x .
$$

Proof. Integration by parts gives us

$$
\left(y, y_{x}\right)=-|y(0)|^{2}-\left(y_{x}, y\right), \quad \text { i.e., } \quad 2 \operatorname{Re}\left(y, y_{x}\right)=-|y(0)|^{2} .
$$

Therefore

$$
\begin{aligned}
\frac{1}{2}|y(0)|^{2}+\operatorname{Re} \lambda\|y\|^{2} & =-\operatorname{Re}(y, F) \leq\|y\|\|F\| \\
& \leq \frac{\alpha}{2} \operatorname{Re} \lambda\|y\|^{2}+\frac{1}{2 \alpha} \frac{\|F\|^{2}}{\operatorname{Re} \lambda}, \quad \alpha>0 .
\end{aligned}
$$

With $\alpha=2$ the first inequality follows. With $\alpha=1$ the second inequality follows.

Lemma A.2. The solution of

$$
y_{x}=-\lambda y+F, \quad y(0)=g, \quad \operatorname{Re} \lambda>0, \quad 0 \leq x<\infty,
$$

satisfies

$$
\|y\|^{2} \leq \frac{1}{\operatorname{Re} \lambda}|g|^{2}+\frac{1}{(\operatorname{Re} \lambda)^{2}}\|F\|^{2} .
$$

Proof.

$$
\langle y, y\rangle_{x}=2 \operatorname{Re}\left\langle y, y_{x}\right\rangle=-2(\operatorname{Re} \lambda)|y|^{2}+2 \operatorname{Re}\langle y, F\rangle .
$$

As $y \in \mathrm{L}^{2}$, integrating we have

$$
\begin{aligned}
-|y(0)|^{2} & =-2 \operatorname{Re} \lambda\|y\|^{2}+2 \operatorname{Re}(y, F) \\
& \leq-2 \operatorname{Re} \lambda\|y\|^{2}+2\|y\|\|F\| \\
& \leq-2 \operatorname{Re} \lambda\|y\|^{2}+\operatorname{Re} \lambda\|y\|^{2}+\frac{\|F\|^{2}}{\operatorname{Re} \lambda} .
\end{aligned}
$$

Thus,

and the lemma follows.

$$
\operatorname{Re} \lambda\|y\|^{2} \leq|y(0)|^{2}+\frac{\|F\|^{2}}{\operatorname{Re} \lambda}
$$

Lemma A.3. Let $a, b$ be real and consider $\sqrt{a+\mathrm{i} b}$ with $-\pi<\arg (a+\mathrm{i} b) \leq \pi, \arg \sqrt{z}=\frac{1}{2} \arg z$. Then, the following inequalities hold

$$
\begin{aligned}
& 2^{-1 / 4} \sqrt{|a|+|b|} \leq|\sqrt{a+\mathrm{i} b}| \leq \sqrt{|a|+|b|} \\
& 2^{-3 / 4}|\sqrt{a+\mathrm{i} b}| \leq 2^{-3 / 4} \sqrt{|a|+|b|} \leq \operatorname{Re} \sqrt{a+\mathrm{i} b} \leq|\sqrt{a+\mathrm{i} b}| \quad \text { for } \quad a \geq 0, \\
& 2^{-5 / 4} \frac{|b|}{|\sqrt{a+\mathrm{i} b}|} \leq 2^{-1} \frac{|b|}{\sqrt{|a|+|b|}} \leq \operatorname{Re} \sqrt{a+\mathrm{i} b} \leq|\sqrt{a+\mathrm{i} b}| \quad \text { for } \quad a \leq 0 .
\end{aligned}
$$


Proof. In polar notation $a+\mathrm{i} b=\rho \mathrm{e}^{\mathrm{i} \theta}, \rho=\sqrt{a^{2}+b^{2}}>0,-\pi<\theta \leq \pi$, and

$$
\sqrt{a+\mathrm{i} b}=\sqrt{\rho} \mathrm{e}^{\mathrm{i} \frac{\theta}{2}}
$$

We have

$$
\sqrt{|a|+|b|}=\sqrt{\rho} \sqrt{|\cos \theta|+|\sin \theta|} \geq 2^{1 / 4} \sqrt{\rho}
$$

and the first inequality in (A.3) follows. The second inequality in (A.3) follows from the triangle inequality. If $a \geq 0$ then $\frac{\theta}{2} \in[-\pi / 4, \pi / 4]$ and (A.3) implies

$$
|\sqrt{a+\mathrm{i} b}| \geq \operatorname{Re} \sqrt{a+\mathrm{i} b}=\sqrt{\rho} \cos (\theta / 2) \geq \frac{\sqrt{2}}{2} \rho \geq 2^{-3 / 4} \sqrt{|a|+|b|} \geq 2^{-3 / 4}|\sqrt{a+\mathrm{i} b}|
$$

which is (A.4). To prove (A.5) notice that, as $a \leq 0$,

$$
\frac{|b|}{\sqrt{|a|+|b|}} \leq \frac{|b|}{|\sqrt{a+\mathrm{i} b}|}=\frac{\rho \sin \theta}{\sqrt{\rho}}=\sqrt{\rho} 2 \sin (\theta / 2) \cos (\theta / 2) \leq 2 \operatorname{Re} \sqrt{a+\mathrm{i} b}
$$

therefore

$$
\frac{1}{2} \frac{|b|}{\sqrt{|a|+|b|}} \leq \operatorname{Re} \sqrt{a+\mathrm{i} b} \leq|\sqrt{a+\mathrm{i} b}|
$$

and the inequality follows from (A.3).

For the forthcoming lemmata, we remind the reader that $s=\eta+\mathrm{i} \xi$, where $\eta, \xi \in \mathbb{R}$, and $\kappa=\sqrt{\omega^{2}+s^{2}}$. We shall now apply the last lemma to

$$
\kappa=\sqrt{\omega^{2}+\eta^{2}-\xi^{2}+2 \mathrm{i} \xi \eta}, \quad \text { i.e., } \quad a=\omega^{2}+\eta^{2}-\xi^{2}, b=2 \xi \eta .
$$

In the following three lemmas we denote by $\delta$ a constant with $0<\delta<1$.

Lemma A.4. Let

$$
\delta_{1}=2^{-1 / 4} \sqrt{\delta}, \quad \delta_{2}=2^{1 / 2}(1-\delta)^{1 / 4}, \quad \delta_{3}=\min \left(\delta_{1}, \delta_{2}\right) .
$$

Then

$$
|\kappa| \geq \begin{cases}\delta_{1} \sqrt{\omega^{2}+|s|^{2}} & \text { if }\left|\omega^{2}+\eta^{2}-\xi^{2}\right| \geq \delta\left(\omega^{2}+|s|^{2}\right) \\ \delta_{2} \sqrt{\sqrt{|s|^{2}+\omega^{2}} \eta} & \text { otherwise. }\end{cases}
$$

Also, always

$$
|\kappa| \geq \delta_{3} \eta
$$

Proof. By (A.3) we obtain, for the first case,

$$
|\kappa| \geq 2^{-1 / 4} \sqrt{\left|\omega^{2}+\eta^{2}-\xi^{2}\right|+2|\xi| \eta} \geq 2^{-1 / 4} \sqrt{\delta} \sqrt{\omega^{2}+|s|^{2}} .
$$

If $\left|\omega^{2}+\eta^{2}-\xi^{2}\right|<\delta\left(\omega^{2}+|s|^{2}\right)$, then

$$
2 \xi^{2} \geq(1-\delta)\left(\omega^{2}+\xi^{2}+\eta^{2}\right)=(1-\delta)\left(\omega^{2}+|s|^{2}\right) .
$$

Therefore

$$
|\kappa| \geq 2^{-1 / 4} \sqrt{2|\xi| \eta} \geq \sqrt{2 \sqrt{1-\delta} \sqrt{\omega^{2}+|s|^{2}} \eta} .
$$

Also $\sqrt{\omega^{2}+|s|^{2}} \geq \eta$ implies (A.7). This proves the lemma. 


\section{Lemma A.5.}

$$
\begin{aligned}
& \operatorname{Re} \kappa \geq \begin{cases}2^{-5 / 4}|\kappa| & \text { if } \omega^{2}+\eta^{2}-\xi^{2} \geq 0, \\
2^{-3 / 4} \frac{\sqrt{\omega^{2}+|s|^{2}}}{|\kappa|} \eta & \text { if } \omega^{2}+\eta^{2}-\xi^{2}<0 .\end{cases} \\
& \operatorname{Re} \kappa \geq \delta_{4} \eta, \quad \delta_{4}=2^{-3 / 4} \min \left(1, \delta_{3}\right) .
\end{aligned}
$$

Proof. If $\omega^{2}+\eta^{2}-\xi^{2} \geq 0$, then (A.4) gives us

$$
\operatorname{Re} \kappa \geq 2^{-3 / 4}|\kappa| \geq 2^{-3 / 4} \delta_{3} \eta .
$$

If $\omega^{2}+\eta^{2}-\xi^{2}<0$, then $2 \xi^{2} \geq \omega^{2}+\eta^{2}+\xi^{2}=\omega^{2}+|s|^{2}$. Therefore (A.5) gives us

$$
\operatorname{Re} \kappa \geq 2^{-5 / 4} \frac{2|\xi| \eta}{|\kappa|} \geq 2^{-3 / 4} \frac{\sqrt{\omega^{2}+|s|^{2}}}{|\kappa|} \eta \geq 2^{-3 / 4} \eta
$$

This proves the lemma.

\section{Lemma A.6.}

$$
|\kappa| \operatorname{Re} \kappa \geq \delta_{6} \sqrt{\omega^{2}+|s|^{2}} \eta, \quad \delta_{6}=\min \left(\delta_{1} \delta_{4}, 2^{-5 / 4} \delta_{2}^{2}, 2^{-3 / 4}\right) .
$$

Proof. If $\omega^{2}+\eta^{2}-\xi^{2} \geq 0$, and $\left|\omega^{2}+\eta^{2}-\xi^{2}\right| \geq \delta\left(\omega^{2}+|s|^{2}\right)$, then (A.6) and (A.8) give us

$$
|\kappa| \operatorname{Re} \kappa \geq \delta_{1} \sqrt{\omega^{2}+|s|^{2}} \delta_{4} \eta .
$$

If $\omega^{2}+\eta^{2}-\xi^{2} \geq 0$, and $\left|\omega^{2}+\eta^{2}-\xi^{2}\right|<\delta\left(\omega^{2}+|s|^{2}\right)$, then (A.6) and (A.8) give us

$$
|\kappa| \operatorname{Re} \kappa \geq 2^{-5 / 4}|\kappa|^{2} \geq 2^{-5 / 4} \delta_{2}^{2} \sqrt{\omega^{2}+|s|^{2}} \eta .
$$

Finally, if $\omega^{2}+\eta^{2}-\xi^{2}<0$, by (A.8) we obtain

$$
|\kappa| \operatorname{Re} \kappa \geq 2^{-3 / 4} \sqrt{|\omega|^{2}+|s|^{2}} \eta .
$$

This proves the lemma.

Lemma A.7. Assume that, for the boundary condition (1), $a>0,|b|<1$. Then there is a constant $\delta>0$ such that, for all $\omega$ and $s$ with $\operatorname{Re} s \geq 0$,

$$
|s+a \kappa-\mathrm{i} b \omega| \geq \delta \sqrt{|s|^{2}+|\omega|^{2}} .
$$

For the proof, see Lemma 3 of [5].

Finally we have a lemma similar to Lemmas A.4 and A.6 but for the normalized variables

$$
\kappa^{\prime}=\sqrt{\eta^{\prime 2}-\xi^{\prime 2}+\omega^{\prime 2}+2 \mathrm{i} \xi^{\prime} \omega^{\prime}}, \quad \xi^{\prime 2}+\omega^{\prime 2}=1,\left|\eta^{\prime}\right| \ll 1 .
$$

By Lemma A.3,

$$
\begin{aligned}
\left|\kappa^{\prime}\right| & \geq 2^{-1 / 4} \sqrt{\left|-\xi^{\prime 2}+\omega^{\prime 2}+\eta^{\prime 2}\right|+2\left|\xi^{\prime}\right|\left|\eta^{\prime}\right|} \\
\operatorname{Re} \kappa^{\prime} & \geq 2^{-3 / 4} \sqrt{\left|-\xi^{\prime 2}+\omega^{\prime 2}+\eta^{\prime 2}\right|+2\left|\xi^{\prime}\right|\left|\eta^{\prime}\right|} \quad \text { if } \xi^{\prime 2} \leq \omega^{\prime 2}+\eta^{\prime 2}, \\
\operatorname{Re} \kappa^{\prime} & \geq \frac{\left|\xi^{\prime}\right| \eta^{\prime}}{\sqrt{\left|-\xi^{\prime 2}+\omega^{\prime 2}+\eta^{\prime 2}\right|+2\left|\xi^{\prime}\right|\left|\eta^{\prime}\right|}} \quad \text { if } \xi^{\prime 2}>\omega^{\prime 2}+\eta^{\prime 2} .
\end{aligned}
$$


Lemma A.8. There is a constants $\delta>0$ such that

$$
\left|\operatorname{Re} \kappa^{\prime}\right| \geq \delta \eta^{\prime}, \quad\left|\kappa^{\prime}\right| \geq 2^{-1 / 4} \eta^{\prime}, \quad\left|\kappa^{\prime}\right|\left|\operatorname{Re} \kappa^{\prime}\right| \geq \delta_{6} \eta^{\prime} .
$$

where $\delta_{6}$ is that of Lemma A.6.

Proof. From Lemma A.6, dividing by $\omega^{2}+\xi^{2}$,

$$
\left|\kappa^{\prime}\right|\left|\operatorname{Re} \kappa^{\prime}\right|=\frac{|\kappa| \operatorname{Re} \kappa}{\omega^{2}+\xi^{2}} \geq \delta_{6} \frac{\sqrt{\omega^{2}+|s|^{2}}}{\sqrt{\omega^{2}+\xi^{2}}} \frac{\eta}{\sqrt{\omega^{2}+\xi^{2}}} \geq \delta_{6} \eta^{\prime} .
$$

Now if $\omega^{\prime 2}-\xi^{\prime 2} \geq 0$, then $\omega^{\prime 2}-\xi^{\prime 2}+\eta^{\prime 2} \geq \eta^{\prime 2}$, and

$$
\sqrt{\left|\omega^{\prime 2}-\xi^{\prime 2}+\eta^{\prime 2}\right|+2\left|\xi^{\prime}\right| \eta^{\prime}} \geq \sqrt{\eta^{\prime 2}+2\left|\xi^{\prime}\right| \eta^{\prime}} \geq \eta^{\prime}
$$

so that, by Lemma A.3,

$$
\left|\kappa^{\prime}\right| \geq 2^{-1 / 4} \eta^{\prime}, \quad \text { and } \quad \operatorname{Re} \kappa^{\prime} \geq 2^{-3 / 4} \eta^{\prime} .
$$

If $\omega^{\prime 2}-\xi^{\prime 2} \leq 0$ then $\left|\xi^{\prime 2}\right| \geq 1 / 2$. From (A.9)

$$
\left|\kappa^{\prime}\right| \geq 2^{-1 / 4} \sqrt{\sqrt{2} \eta^{\prime}} \geq \eta^{\prime} .
$$

This last estimate also holds for $\left|\operatorname{Re} \kappa^{\prime}\right|$ when $\omega^{\prime 2}-\xi^{\prime 2}+\eta^{\prime 2} \geq 0$. When $\omega^{\prime 2}-\xi^{\prime 2}+\eta^{\prime 2} \leq 0$ we just use $\left|\omega^{\prime 2}-\xi^{\prime 2}+\eta^{\prime 2}\right|+2\left|\xi^{\prime}\right| \eta^{\prime} \leq$ const. and from (A.9) the estimate for Re $\kappa^{\prime}$ follows from (A.9). This proves the lemma.

Acknowledgements. We thank Prof. Oscar Reula who participated in the original discussions leading to this work.

\section{REFERENCES}

[1] M.S. Agranovich, Theorem on matrices depending on parameters and its applications to hyperbolic systems. Funct. Anal. Appl. 6 (1972) 85-93.

[2] B. Gustafsson, H.-O. Kreiss and J. Oliger, Time dependent problems and difference methods. Wiley-Interscience (1995).

[3] H.-O. Kreiss, Initial boundary value problems for hyperbolic systems. Comm. Pure Appl. Math. 23 (1970) $277-298$.

[4] H.-O. Kreiss and J. Lorenz, Initial-boundary value problems and the Navier-Stokes equations. Academic Press, San Diego (1989).

[5] H.-O. Kreiss and J. Winicour, Problems which are well posed in a generalized sense with applications to the Einstein equations. Class. Quantum Grav. 23 (2006) 405-420. 